\title{
Article \\ How Different Fermentation Type Affects Volatile Composition of Plum Jerkums
}

\author{
Magdalena Januszek*(D) and Paweł Satora \\ Department of Fermentation Technology and Microbiology, Faculty of Food Technology, \\ University of Agriculture in Kraków, Balicka Street 122, 30-149 Kraków, Poland; pawel.satora@urk.edu.pl \\ * Correspondence: magdalena.januszek@urk.edu.pl
}

check for

updates

Citation: Januszek, M.; Satora, P. How Different Fermentation Type Affects Volatile Composition of Plum Jerkums. Appl. Sci. 2021, 11, 4658. https://doi.org/10.3390/app11104658

Academic Editors: Ilda Caldeira and Mar Vilanova

Received: 4 March 2021

Accepted: 17 May 2021

Published: 19 May 2021

Publisher's Note: MDPI stays neutral with regard to jurisdictional claims in published maps and institutional affiliations.

Copyright: (c) 2021 by the authors. Licensee MDPI, Basel, Switzerland. This article is an open access article distributed under the terms and conditions of the Creative Commons Attribution (CC BY) license (https:// creativecommons.org/licenses/by/ $4.0 /)$.

\begin{abstract}
Quality of plum jerkum is significantly associated to the profile of volatile compounds. Therefore, we decided to assess the impact of various fermentation types on selected properties of plum jerkums, especially compounds which contribute to the aroma of the finished product. We used the following yeast strains: S. cerevisiae S1, H. uvarum H2, and Ethanol RED (S. cerevisiae). Moreover, we considered spontaneous fermentation. S. cerevisiae and H. uvarum strains were isolated during the fermentation of Čačanska Lepotica or Węgierka Dabrowicka (plum cultivars), respectively. As for fermentation type, spontaneous fermentation of $H$. uvarum $\mathrm{H} 2$ provided the best results. It could be associated to the fact that plum juices fermented with $H$. uvarum $\mathrm{H} 2$ presented the highest concentration of terpenoids, esters, or some higher alcohols. In the current paper, application of indigenous strains of yeasts resulted in the required oenological characteristics, e.g., highest fermentation efficiency and concentration of ethanol was determined in juices fermented with Ethanol RED (S. cerevisiae) and also with S. cerevisiae S1. Our results suggested that indigenous strains of yeasts present in plums demonstrate great potential for the production of plum jerkums of high quality.
\end{abstract}

Keywords: plum jerkums; Saccharomyces cerevisiae; Hanseniaspora uvarum; volatile compounds

\section{Introduction}

Oenological parameters, volatile compounds, and overall quality of fruit wines depend on the impact of various factors, such as quality of raw materials, mainly their chemical composition, primary processing techniques, fermentation conditions, further vinification procedures, and possibly aging. Volatile compounds in alcoholic beverages produced with various fruits have a very diverse origin. Some of them naturally occur in fruits and could be exposed to various transformations carried out by microorganisms during fermentation or aging and oxidation. Other aroma compounds are produced by microorganisms, e.g., esters, volatile acids, higher alcohols, carbonyl compounds, and some terpenoids. However, chemical composition of fruits is a key factor to fruit wine quality [1-3].

Plum (Prunus domestica) could be used for winemaking or for cider-like alcoholic beverages-plum jerkum is produced in Central England. Plum wine, which is particularly popular in Germany and Pacific Coastal states, has an appealing color and is high quality. Plum could be tart and sweet at the same time which originates from the concentration of sugars and acids. The skin itself might be particularly tart [3].

Fresh plums contain, e.g., ethyl acetate, 2-methyl-1-propyl acetate, propyl acetate, 3-methylbutyl acetate, butyl acetate, pentyl acetate, 3-hexenyl acetate, 1-hexanol, (Z)-3hexenol, nonanal, linalool, benzaldehyde, $\gamma$-octalactone, $\gamma$-decalactone, and others which contribute to the aroma of alcoholic beverages [4]. Presence of these substances gives them a fresh, fruity, and floral aroma.

Microorganisms which carry out alcoholic fermentation are either indigenous microorganisms present on fruits or added starter cultures. Initially, in spontaneous fermentation, 
musts are dominated by yeast which belong to Kloeckera/Hanseniaspora, and Candida genera. In the middle stages of fermentation when the concentration of ethyl alcohol rises to 3-4\%, mainly species of Pichia and Metschnikowia are present [5]. After that, when ethanol concentration is high, Saccharomyces yeast dominates fermentation. However, in the majority of cases, wine producers use starter cultures of $S$. cerevisiae. This approach enables obtaining wine of consistent quality [5]. Saccharomyces yeast could produce various volatile compounds-esters, especially ethyl acetate, diethyl succinate, ethyl caproate, ethyl caprylate, methyl anthranilate, aldehydes, ketones, and terpenes, e.g., linalool, linalool oxide, guaiacol, $\beta$-ionone, and citral. It also provides high sensory quality of alcoholic beverages $[1,6]$.

H. uvarum could produce increased amounts of volatiles that improve fruit wine quality such as higher alcohols, esters, and carbonyl compounds. Therefore, mixed cultures of $S$. cerevisiae and $H$. uvarum could be used for the production of fruit wine with improved aroma and quality. Among wild yeast, H. uvarum strains could synthesize a significant quantity of acetic acid and ethyl acetate which could spoil wine quality when in excess [7].

The current study mostly focuses on assessing the impact of microbial fermentation on chemical composition and the volatile profile of plum jerkums. For the fermentation, spontaneous fermentation, S. cerevisiae S1, H. uvarum H2, and Ethanol RED (S. cerevisiae) were used. S. cerevisiae and H. uvarum strains were isolated during the fermentation of Čačanska Lepotica or Wegierka Dabrowicka, respectively. In this study, four cultivars of plums were used: Stanley, Wegierka Zwykła, Wegierka Dabrowicka, and Čačanska Lepotica. The first three of them are commonly cultivated in Poland and used for processing in the food industry [8], while Čačanska lepotica has great potential for fruit wine production [9].

\section{Materials and Methods}

\subsection{Fermentation of Plum Mashes}

Plum musts used for the fermentation were obtained from Wegierka Zwykła, Wegierka Dąbrowicka, Stanley, and Čačanska Lepotica cultivars harvested in Łacko $\left(49^{\circ} 33^{\prime} 30^{\prime \prime} \mathrm{N}\right.$ $20^{\circ} 26^{\prime} 06^{\prime \prime}$ E, Małopolska district, Poland). After washing, we manually crushed plums, applied pectynolytic preparation Pektopol PT-400 $(0.5 \mathrm{~mL} / \mathrm{kg})$ for $12 \mathrm{~h}$ at $20{ }^{\circ} \mathrm{C}$, and weighed $2 \mathrm{~kg}$ portions which were transferred to $3 \mathrm{~L}$ sterile glass flasks. Except for variants designated for spontaneous fermentation, samples were pasteurized at $80{ }^{\circ} \mathrm{C}$ for $10 \mathrm{~min}$ and inoculated ( $0.3 \mathrm{~g} \mathrm{d.w./L} \mathrm{of} \mathrm{musts)} \mathrm{with} \mathrm{different} \mathrm{types} \mathrm{of} \mathrm{yeast} \mathrm{or} \mathrm{fermented} \mathrm{spontaneously.}$ We used the Hanseniaspora uvarum $\mathrm{H} 2$ (MN464119) strain isolated during the second day of fermentation of Wegierka Dabrowicka fruits; Saccharomyces cerevisiae S1 (MN464134) isolated on the 30th day of fermentation of Čačanska Lepotica [10]; and commercial distiller's yeast RED Ethanol (Saccharomyces cerevisiae, Starowar, Warsaw, Poland). H. uvarum and $S$. cerevisiae were inoculated on slants of Sabouraud agar incubated for $24 \mathrm{~h}$ at $28{ }^{\circ} \mathrm{C}$. Then colonies were transferred to $10 \mathrm{~mL}$ of Sabouraud broth and incubated for another $24 \mathrm{~h}$ at $28{ }^{\circ} \mathrm{C}$. Finally, yeast suspension was transferred to $140 \mathrm{~mL}$ of fresh Sabouraud broth and incubated $\left(24 \mathrm{~h} / 28^{\circ} \mathrm{C}\right)$. Yeast suspension was centrifuged $(5000 \mathrm{rpm} / 15 \mathrm{~min})$, supernatant was removed, and the pellet was resuspended in the plum must. We stopped flasks with a plug equipped with the fermentation tube. Then tubes were filled with glycerol. Flasks were stored under a controlled temperature $\left(20^{\circ} \mathrm{C}\right)$. Changes of weight for each flask was recorded each day. Fermentation was carried out until the end of changes in mass loss associated with $\mathrm{CO}_{2}$ release. When fermentation was complete, samples of fermented musts were collected and stored $\left(-20^{\circ} \mathrm{C}\right)$ for chemical analysis.

\subsection{Chemical Composition of Fresh and Fermented Plum Mashes}

The concentration of ethanol, reducing sugars, sugar-free extract, total extract, and sucrose concentrations and titratable acidity were determined using officially approved methods described by the International Organization of Vine and Wine (OIV) [11]. Titratable acidity (TA) was calculated from the results obtained with Mettler DL 25 titrator (Mettler Toledo, Greifensee, Switzerland). We used 0.1 M NaOH as a titrant. Samples were 
titrated to $\mathrm{pH}$ 7.0. Results were shown as grams of malic acid per liter. For the calculation of fermentation efficiency, we applied theoretical correlations which were as follows- $1 \mathrm{~g}$ of reducing sugars or sucrose could be transformed to $0.511 \mathrm{~g}$ or $0.538 \mathrm{~g}$ of ethanol, respectively. Ninhydrin method was used for the determination of free amino nitrogen (FAN). Then, $2 \mathrm{~mL}$ of diluted $(\times 50)$ plum musts or jerkums $(1 \mathrm{~mL})$ were transferred to caped glass tubes. Then, we added $1 \mathrm{~mL}$ of ninhydrin reagent and heated tubes in the boiling water bath for $16 \mathrm{~min}$. After cooling samples, we added $5 \mathrm{~mL}$ of dilution reagent. Finally, the absorbance was measured at $575 \mathrm{~nm}$ against distilled water with ninhydrin as a blank [12].

\subsection{Determination of Sugar and Main Organic Acids Content by High-Performance Liquid Chromatography (HPLC)}

Centrifuged (MPW-65R, MPW Med. Instruments, Warszawa, Poland-14,000 $\times \mathrm{g} / 5 \mathrm{~min}$ ) musts or jerkums were diluted $(\times 5)$ with deionized water. Then we used syringe filters ( $0.45 \mu \mathrm{m}$ pore density, Sartorius AG, Getinge, Germany) to filtrate obtained dilutions. We analyzed organic acids on a Shimadzu NEXERA XR chromatograph (Kyoto, Japan). That piece of equipment has a pump system, and a UV/Vis detector (monitored at $210 \mathrm{~nm}$ ). Malic, succinic, acetic, tartaric, lactic, and citric acids (Sigma-Aldrich, St. Louis, MO, USA) were analyzed using Rezex ROA-Organic Acid Aminex HPX-87H column $(300 \times 7.8 \mathrm{~mm})$ (Rezex, Torrance, CA, USA). Isocrating elutions of samples was carried out at $40{ }^{\circ} \mathrm{C}$ with $0.005 \mathrm{M} \mathrm{H}_{2} \mathrm{SO}_{4}$ ) at a flow rate of $0.4 \mathrm{~mL} / \mathrm{min}$. We analyzed sugar profile with the same piece of equipment, however, we replaced the UV/Vis detector with a refractometer detector RF-20A. Sugars were separated on an Asahipak NH2P-50, $4.6 \times 250 \mathrm{~mm}$ Shodex column (Showa Denko America, Munich, Germany) at $30^{\circ} \mathrm{C}$. The isocratic elution program $(0.8 \mathrm{~mL} / \mathrm{min})$ lasted $16 \mathrm{~min}$. We used an aqueous solution of acetonitrile $(70 \%)$ for elution. We prepared standard curves for each tested compound: fructose, glucose, sucrose, and glycerol.

\subsection{Volatile Composition of Fresh and Fermented Plum Mashes Using Solid Phase Microextraction-Gas Chromatography-Mass Spectrometry (SPME-GC-MS) and Gas Chromatography-Flame Ionization Detector (GC-FID) Methods}

Analysis of volatile compounds was performed using gas chromatography with mass spectrometry [1,6]. We suspended $0.05 \mathrm{mg}$ of 4-methyl-2-pentanol/L and $0.5 \mu \mathrm{g}$ of ethyl nonanoate, (Sigma-Aldrich, St. Louis, MO, USA) in $1 \mathrm{~mL}$ saturated saline and that solution was used as an internal standard. We transferred $1 \mathrm{~mL}$ of juice or jerkums to a $10 \mathrm{~mL}$ vial. We conditioned the SPME device (Supelco Inc., Bellefonte, PA, USA, $100 \mu \mathrm{m}$ polydimethylsiloxane fiber) in the GC injector port at $250{ }^{\circ} \mathrm{C}$ for $1 \mathrm{~h}$. After inserting the fiber in the glass vials, samples were stirred $\left(300 \mathrm{rpm} / 40{ }^{\circ} \mathrm{C} / 30 \mathrm{~min}\right)$ and then fibers were transferred to the injector port Agilent Technologies $7890 \mathrm{~B}$ chromatograph system (Agilent Technologies, Santa Clara, CA, USA). The system is equipped with an LECO Pegasus HT, High Throughput time-of-flight mass spectrometry (TOFMS) detector. Fibers were held for $3 \mathrm{~min}$ in the inlet. Transfers of the fiber were automated with GERSTEL MultiPurpose Sampler (MPS). Volatile compounds were separated on at the Rtx-1ms capillary column (Crossbond 100\% dimethyl polysiloxane, $30 \mathrm{~m} \times 0.53 \mathrm{~mm} \times 0.5 \mu \mathrm{m}$ ). The injector and detector were heated to $250{ }^{\circ} \mathrm{C}$, while the separation of compounds was initiated at $40{ }^{\circ} \mathrm{C} / 3 \mathrm{~min}$ and then the temperature increased at an increment of $8{ }^{\circ} \mathrm{C} / \mathrm{min}$ to $230^{\circ} \mathrm{C}$. Finally, samples were held at maximum temperature for $9 \mathrm{~min}$. Helium, which was used as a carrier gas, was delivered at a constant flow $(1.0 \mathrm{~mL} / \mathrm{min})$, while EIMS electron energy was $70 \mathrm{eV}$. Temperature of the source and connection parts was $250{ }^{\circ} \mathrm{C}$. Analyte were transferred in the splitless mode. The mass spectrometer detector (MSD) was set to the scan mode from $\mathrm{m} / z=40$ to $\mathrm{m} / z=400$. We identified volatiles using mass spectral libraries and linear retention indices (calculated from a series of $n$-alkanes from C6 to C30). Semi-quantitative analysis of substances was assessed from the ratio of relative peak area of each identified component, to relative peak area of adequate internal standard (ethyl nonanoate for esters, anethol for terpenoids, and 4-methyl-2-pentanol for other components). Obtained results were analyzed in the National Institute of Standards and 
Technology (NIST) database. This method was validated based on the method described by Antalick et al., 2010 [13].

Determination of the selected volatiles was analyzed using gas chromatography (Hewlett Packard 5890 Series II chromatograph system; Agilent Technologies, Santa Clara, CA, USA) with a flame ionization detector as described by Satora and Tuszyński [3]. We used HP-INNOWax capillary column ( $30 \mathrm{~m} \times 0.53 \mathrm{~mm}$ ID with $1.0 \mu \mathrm{m}$ film thickness, crosslinked polyethylene glycol stationary phase; Agilent, Santa Clara, CA, USA) for the separation of volatile compounds. The temperature of both detector and injector was set to $250{ }^{\circ} \mathrm{C}$. Initial temperature of the column was $35^{\circ} \mathrm{C}(5 \mathrm{~min})$ and then it was increased to $110^{\circ} \mathrm{C}$ at an increment of $5^{\circ} \mathrm{C} / \mathrm{min}$, and then it was increased to $220^{\circ} \mathrm{C}$ at an increment of $40^{\circ} \mathrm{C} / \mathrm{min}$. The last temperature was sustained for $3 \mathrm{~min}$. We used helium as a carrier gas $(20.0 \mathrm{~mL} / \mathrm{min})$. Hydrogen was delivered at a $33.0 \mathrm{~mL} / \mathrm{min}$ flow speed, while for air it was $400 \mathrm{~mL} / \mathrm{min}$. Volatiles were identified and quantified (acetaldehyde, acetone, methanol, propanol, isobutanol, butanol, pentanol, hexanol, phenylethanol, amyl alcohols, and ethyl acetate, Sigma-Aldrich) by the comparison of obtained surface peak area to those obtained for standards.

\subsection{Statistical Analysis}

Each experiment was carried out in three physical replicates. All analyses were carried out for each replicate. We used R 3.6.1 (Vienna, Austria) for statistical analysis. The ShapiroWilk test was applied to assess the normality of the data distribution. Multivariate analysis of variance (MANOVA) was carried out prior to the post hoc Tukey test.

\section{Results and Discussion}

\subsection{Chemical Composition of Fresh Plum Musts and Jerkums}

Musts obtained from Węgierka Dabrowicka and Stanley demonstrated the highest concentration of total extract and total sugars which constituted about $80 \%$ of the total extract. However, in comparison to other cited studies, concentrations of total sugars in our samples were slightly low and ranged from 113.2 (Węgierka Zwykła) to $123.8 \mathrm{~g} / \mathrm{L}$ (Wegierka Dabrowicka) (Table 1), whereas in other studies it reached 153.6 g/L [14] or $127.5 \mathrm{~g} / \mathrm{L}$ [4], respectively. Sugar content in plum influences consumer perception of maturity of consumed fruits and its concentration depends on the fruit cultivar, climatic conditions, and harvesting time [4,15].

Table 1. Chemical composition of fresh plum musts.

\begin{tabular}{|c|c|c|c|c|c|c|c|c|c|c|}
\hline $\begin{array}{l}\text { Plum } \\
\text { Cultivars }\end{array}$ & $\begin{array}{c}\text { Total } \\
\text { Extract }\end{array}$ & $\begin{array}{l}\text { Total } \\
\text { Sugars }\end{array}$ & $\begin{array}{c}\text { Reducing } \\
\text { Sugars }\end{array}$ & Sucrose & $\begin{array}{c}\text { Sugar-Free } \\
\text { Extract }\end{array}$ & $\begin{array}{c}\text { Titratable } \\
\text { Acidity }\end{array}$ & $\begin{array}{l}\text { Citric } \\
\text { Acid }\end{array}$ & $\begin{array}{l}\text { Malic } \\
\text { Acid }\end{array}$ & $\begin{array}{l}\text { Succinic } \\
\text { Acid }\end{array}$ & $\begin{array}{c}\text { Free Amino } \\
\text { Nitrogen }\end{array}$ \\
\hline & & & & {$[\mathrm{g} / \mathrm{L}]$} & & & & & {$[\mathrm{mg} / \mathrm{L}]$} & \\
\hline $\begin{array}{c}\text { Wẹgierka } \\
\text { Zwykła }\end{array}$ & $\begin{array}{l}146.0^{\mathrm{c}} \\
( \pm 2.0)\end{array}$ & $\begin{array}{l}113.2^{\mathrm{b}} \\
( \pm 4.3)\end{array}$ & $\begin{array}{l}42.6^{\mathrm{ab}} \\
( \pm 2.3)\end{array}$ & $\begin{array}{l}51.4^{\mathrm{a}} \\
( \pm 2.0)\end{array}$ & $\begin{array}{l}26.7^{\mathrm{b}} \\
( \pm 4.2)\end{array}$ & $\begin{array}{c}9.06^{\mathrm{b}} \\
( \pm 0.39)\end{array}$ & $\begin{array}{c}1.12^{\mathrm{b}} \\
( \pm 0.07)\end{array}$ & $\begin{array}{c}6.13^{\mathrm{ab}} \\
( \pm 0.24)\end{array}$ & $\begin{array}{c}0.56^{\mathrm{b}} \\
( \pm 0.11)\end{array}$ & $\begin{array}{l}119.6^{c} \\
( \pm 11.4)\end{array}$ \\
\hline $\begin{array}{c}\text { Wẹgierka } \\
\text { Dabrowicka }\end{array}$ & $\begin{array}{l}155.7^{\mathrm{b}} \\
( \pm 1.5)\end{array}$ & $\begin{array}{l}123.8^{\mathrm{a}} \\
( \pm 5.7)\end{array}$ & $\begin{array}{l}45.9^{\mathrm{a}} \\
( \pm 2.6) \\
\end{array}$ & $\begin{array}{l}47.9^{\mathrm{ab}} \\
( \pm 2.9) \\
\end{array}$ & $\begin{array}{l}37.8^{\mathrm{a}} \\
( \pm 4.2)\end{array}$ & $\begin{array}{l}12.22^{\mathrm{a}} \\
( \pm 0.23)\end{array}$ & $\begin{array}{c}1.00^{\mathrm{b}} \\
( \pm 0.04)\end{array}$ & $\begin{array}{c}6.41 \mathrm{ab} \\
( \pm 0.58)\end{array}$ & $\begin{array}{c}1.13^{\mathrm{a}} \\
( \pm 0.14)\end{array}$ & $\begin{array}{c}135.6^{\mathrm{bc}} \\
( \pm 4.6)\end{array}$ \\
\hline Stanley & $\begin{array}{c}161.7^{\mathrm{ab}} \\
( \pm 6.4)\end{array}$ & $\begin{array}{l}121.4^{\mathrm{a}} \\
( \pm 2.6)\end{array}$ & $\begin{array}{l}40.3^{\mathrm{ab}} \\
( \pm 4.3)\end{array}$ & $\begin{array}{l}56.7^{\mathrm{a}} \\
( \pm 2.2)\end{array}$ & $\begin{array}{l}37.7^{\mathrm{a}} \\
( \pm 9.0)\end{array}$ & $\begin{array}{c}9.81^{\mathrm{b}} \\
( \pm 0.47)\end{array}$ & $\begin{array}{c}1.50^{\mathrm{a}} \\
( \pm 0.17)\end{array}$ & $\begin{array}{c}7.26^{\mathrm{a}} \\
( \pm 0.29)\end{array}$ & $\begin{array}{c}0.82^{\mathrm{a}} \\
( \pm 0.24)\end{array}$ & $\begin{array}{l}156.4^{b} \\
( \pm 6.6)\end{array}$ \\
\hline $\begin{array}{l}\text { Čačanska } \\
\text { Lepotica }\end{array}$ & $\begin{array}{l}151.0^{c} \\
( \pm 1.0)\end{array}$ & $\begin{array}{c}119.4^{\mathrm{ab}} \\
( \pm 2.6)\end{array}$ & $\begin{array}{l}32.1^{\mathrm{b}} \\
( \pm 2.0)\end{array}$ & $\begin{array}{l}44.5^{\mathrm{b}} \\
( \pm 1.6)\end{array}$ & $\begin{array}{l}41.4^{\mathrm{a}} \\
( \pm 2.5)\end{array}$ & $\begin{array}{l}11.90^{\mathrm{a}} \\
( \pm 0.53)\end{array}$ & $\begin{array}{c}1.04^{\mathrm{b}} \\
( \pm 0.12)\end{array}$ & $\begin{array}{c}6.56^{\mathrm{ab}} \\
( \pm 0.33)\end{array}$ & $\begin{array}{c}0.98^{\mathrm{a}} \\
( \pm 0.05)\end{array}$ & $\begin{array}{l}212.1^{\mathrm{a}} \\
( \pm 5.1)\end{array}$ \\
\hline Significance & ** & $* * *$ & $* * *$ & $* * *$ & $* * *$ & $* * *$ & $* * *$ & $* * *$ & $* * *$ & $* *$ \\
\hline
\end{tabular}

Values with different superscript roman letters $(\mathrm{a}-\mathrm{c})$ in the same column indicate statistically significant differences at $p<0.05 ; n=5$; $0.001^{* * *} ; 0.01^{* *}$.

Total extract of analyzed samples decreased after fermentation, which was caused by intense consumption of sugars during that process and high fermentation efficiency ranging from 66.6 to $99.3 \%$ (Table 2). The highest fermentation efficiency and concentration of ethanol (more than $7 \%$ vol.) was determined in musts fermented with Ethanol RED 
(S. cerevisiae) or with S. cerevisiae S1, while lowest efficiency was shown for musts fermented spontaneously. According to the manufacturer's specification, Ethanol RED is more resistant to ethanol concentration exceeding $18 \%$ vol. and provides higher fermentation efficiency than many other yeast strains [16]. This would explain the results described in the current paper.

Table 2. Chemical composition of plum jerkums fermented spontaneously or with different yeast starter cultures.

\begin{tabular}{|c|c|c|c|c|c|c|}
\hline $\begin{array}{l}\text { Plum } \\
\text { Cultivars }\end{array}$ & $\begin{array}{c}\text { Type of } \\
\text { Fermentation }\end{array}$ & Total Extract & $\begin{array}{l}\text { Titratable } \\
\text { Acidity }\end{array}$ & Ethanol & $\begin{array}{l}\text { Free Amino } \\
\text { Nitrogen }\end{array}$ & $\begin{array}{c}\text { Fermentation } \\
\text { Efficiency }\end{array}$ \\
\hline & & \multicolumn{2}{|c|}{$[\mathrm{g} / \mathrm{L}]$} & [\% vol.] & {$[\mathrm{mg} / \mathrm{L}]$} & [\%] \\
\hline \multirow{4}{*}{$\begin{array}{l}\text { Węgierka } \\
\text { Zwykła }\end{array}$} & S. cerevisiae S1 & $\begin{array}{l}42.0^{\mathrm{ab}} \\
( \pm 2.2)\end{array}$ & $\begin{array}{c}3.72 \mathrm{e} \\
( \pm 0.12)\end{array}$ & $\begin{array}{c}6.9^{\mathrm{b}} \\
( \pm 0.2)\end{array}$ & $\begin{array}{l}46.29 \mathrm{~d} \\
( \pm 1.98)\end{array}$ & $\begin{array}{l}93.8^{a b} \\
( \pm 1.2)\end{array}$ \\
\hline & H. uvarum $\mathrm{H} 2$ & $\begin{array}{l}44.7^{\mathrm{ab}} \\
( \pm 1.2)\end{array}$ & $\begin{array}{c}3.63^{\mathrm{e}} \\
( \pm 0.21)\end{array}$ & $\begin{array}{c}6.8^{\mathrm{b}} \\
( \pm 0.1)\end{array}$ & $\begin{array}{l}51.67 \mathrm{~cd} \\
( \pm 2.18)\end{array}$ & $\begin{array}{l}92.3^{\mathrm{ab}} \\
( \pm 2.1)\end{array}$ \\
\hline & $\begin{array}{l}\text { Spontaneous } \\
\text { fermentation }\end{array}$ & $\begin{array}{l}39.0^{\mathrm{ab}} \\
( \pm 0.8)\end{array}$ & $\begin{array}{c}3.73^{\mathrm{e}} \\
( \pm 0.23)\end{array}$ & $\begin{array}{c}5.7^{\mathrm{c}} \\
( \pm 0.1)\end{array}$ & $\begin{array}{l}52.46^{\mathrm{c}} \\
( \pm 1.02)\end{array}$ & $\begin{array}{l}78.4^{\mathrm{bc}} \\
( \pm 0.9)\end{array}$ \\
\hline & $\begin{array}{l}\text { Ethanol RED } \\
\text { (S. cerevisiae) }\end{array}$ & $\begin{array}{l}45.3^{a b} \\
( \pm 0.5)\end{array}$ & $\begin{array}{c}4.43^{\mathrm{d}} \\
( \pm 0.07)\end{array}$ & $\begin{array}{l}7.2^{\mathrm{ab}} \\
( \pm 0.2)\end{array}$ & $\begin{array}{l}47.46^{\mathrm{d}} \\
( \pm 1.06)\end{array}$ & $\begin{array}{l}97.8^{\mathrm{a}} \\
( \pm 1.4)\end{array}$ \\
\hline \multirow{4}{*}{$\begin{array}{c}\text { Wegierka } \\
\text { Dąbrowicka }\end{array}$} & S. cerevisiae S1 & $\begin{array}{l}36.3^{\mathrm{b}} \\
( \pm 2.1)\end{array}$ & $\begin{array}{c}9.14^{\mathrm{a}} \\
( \pm 0.03)\end{array}$ & $\begin{array}{c}7.9^{\mathrm{a}} \\
( \pm 0.1)\end{array}$ & $\begin{array}{l}57.61^{\mathrm{c}} \\
( \pm 2.61)\end{array}$ & $\begin{array}{l}98.8^{\mathrm{a}} \\
( \pm 1.3)\end{array}$ \\
\hline & H. uvarum $\mathrm{H} 2$ & $\begin{array}{l}33.0^{\mathrm{bc}} \\
( \pm 3.7)\end{array}$ & $\begin{array}{c}6.53^{b} \\
( \pm 1.73)\end{array}$ & $\begin{array}{c}7.8^{\mathrm{a}} \\
( \pm 0.2)\end{array}$ & $\begin{array}{l}45.32 \mathrm{~d} \\
( \pm 1.51)\end{array}$ & $\begin{array}{l}98.6^{\mathrm{a}} \\
( \pm 0.8)\end{array}$ \\
\hline & $\begin{array}{l}\text { Spontaneous } \\
\text { fermentation }\end{array}$ & $\begin{array}{l}48.7^{\mathrm{a}} \\
( \pm 5.8)\end{array}$ & $\begin{array}{c}8.55^{\mathrm{a}} \\
( \pm 0.72)\end{array}$ & $\begin{array}{c}5.4^{c} \\
( \pm 0.3)\end{array}$ & $\begin{array}{l}44.91^{d} \\
( \pm 6.02)\end{array}$ & $\begin{array}{l}67.6^{c} \\
( \pm 2.1)\end{array}$ \\
\hline & $\begin{array}{l}\text { Ethanol RED } \\
\text { (S. cerevisiae) }\end{array}$ & $\begin{array}{l}46.0^{\mathrm{a}} \\
( \pm 0.8)\end{array}$ & $\begin{array}{c}8.33^{\mathrm{a}} \\
( \pm 0.40)\end{array}$ & $\begin{array}{c}8.0^{\mathrm{a}} \\
( \pm 0.3)\end{array}$ & $\begin{array}{l}54.41^{\mathrm{c}} \\
( \pm 3.31) \\
\end{array}$ & $\begin{array}{l}99.3^{\mathrm{a}} \\
( \pm 0.3)\end{array}$ \\
\hline \multirow{4}{*}{ Stanley } & S. cerevisiae S1 & $\begin{array}{l}44.7 \mathrm{ab} \\
( \pm 0.5)\end{array}$ & $\begin{array}{c}5.81^{\mathrm{c}} \\
( \pm 0.13)\end{array}$ & $\begin{array}{l}7.6^{\mathrm{a}} \\
( \pm 0.3)\end{array}$ & $\begin{array}{l}66.33^{b} \\
( \pm 3.03)\end{array}$ & $\begin{array}{l}96.4^{\mathrm{a}} \\
( \pm 0.8)\end{array}$ \\
\hline & H. uvarum $\mathrm{H} 2$ & $\begin{array}{l}43.0^{\mathrm{ab}} \\
( \pm 0.8)\end{array}$ & $\begin{array}{c}6.10^{b} \\
( \pm 0.38)\end{array}$ & $\begin{array}{c}7.4^{\mathrm{a}} \\
( \pm 0.1)\end{array}$ & $\begin{array}{l}59.11^{\mathrm{c}} \\
( \pm 0.61)\end{array}$ & $\begin{array}{l}93.6^{\mathrm{ab}} \\
( \pm 1.0)\end{array}$ \\
\hline & $\begin{array}{l}\text { Spontaneous } \\
\text { fermentation }\end{array}$ & $\begin{array}{l}42.7^{\mathrm{ab}} \\
( \pm 1.2)\end{array}$ & $\begin{array}{c}5.38^{c} \\
( \pm 0.32)\end{array}$ & $\begin{array}{c}6.7^{\mathrm{b}} \\
( \pm 1.1)\end{array}$ & $\begin{array}{l}57.72^{c} \\
( \pm 0.83)\end{array}$ & $\begin{array}{l}84.8^{b} \\
( \pm 0.6)\end{array}$ \\
\hline & $\begin{array}{l}\text { Ethanol RED } \\
\text { (S. cerevisiae) }\end{array}$ & $\begin{array}{l}39.7 \mathrm{ab} \\
( \pm 1.6)\end{array}$ & $\begin{array}{l}7.19^{a b} \\
( \pm 0.41)\end{array}$ & $\begin{array}{c}7.8^{\mathrm{a}} \\
( \pm 0.9)\end{array}$ & $\begin{array}{l}63.32 \mathrm{bc} \\
( \pm 0.54)\end{array}$ & $\begin{array}{l}98.9^{\mathrm{a}} \\
( \pm 1.2)\end{array}$ \\
\hline \multirow{4}{*}{$\begin{array}{l}\text { Čačanska } \\
\text { Lepotica }\end{array}$} & S. cerevisiae S1 & $\begin{array}{l}28.0^{c} \\
( \pm 1.6)\end{array}$ & $\begin{array}{c}5.47^{\mathrm{c}} \\
( \pm 0.41)\end{array}$ & $\begin{array}{c}7.4^{\mathrm{a}} \\
( \pm 0.3)\end{array}$ & $\begin{array}{l}70.65^{\mathrm{a}} \\
( \pm 1.44) \\
\end{array}$ & $\begin{array}{l}97.6^{\mathrm{a}} \\
( \pm 0.6)\end{array}$ \\
\hline & H. uvarum $\mathrm{H} 2$ & $\begin{array}{l}37.3^{b} \\
( \pm 2.1)\end{array}$ & $\begin{array}{c}5.65^{c} \\
( \pm 0.36)\end{array}$ & $\begin{array}{l}7.0^{\mathrm{ab}} \\
( \pm 0.2)\end{array}$ & $\begin{array}{l}69.93^{\mathrm{ab}} \\
( \pm 2.40)\end{array}$ & $\begin{array}{l}91.1^{\mathrm{ab}} \\
( \pm 0.8)\end{array}$ \\
\hline & $\begin{array}{l}\text { Spontaneous } \\
\text { fermentation }\end{array}$ & $\begin{array}{l}53.0^{\mathrm{a}} \\
( \pm 7.4)\end{array}$ & $\begin{array}{l}4.93 \mathrm{~cd} \\
( \pm 0.18)\end{array}$ & $\begin{array}{c}5.1^{\mathrm{c}} \\
( \pm 0.7)\end{array}$ & $\begin{array}{l}72.49^{a} \\
( \pm 1.43)\end{array}$ & $\begin{array}{l}66.6^{c} \\
( \pm 4.3)\end{array}$ \\
\hline & $\begin{array}{l}\text { Ethanol RED } \\
\text { (S. cerevisiae) }\end{array}$ & $\begin{array}{l}46.0^{\mathrm{a}} \\
( \pm 0.8)\end{array}$ & $\begin{array}{c}5.64^{\mathrm{c}} \\
( \pm 0.12)\end{array}$ & $\begin{array}{c}7.5^{\mathrm{a}} \\
( \pm 0.2)\end{array}$ & $\begin{array}{l}73.65^{a} \\
( \pm 3.89)\end{array}$ & $\begin{array}{l}97.7^{\mathrm{a}} \\
( \pm 1.1)\end{array}$ \\
\hline \multicolumn{2}{|c|}{ Significance } & $* *$ & * & $* * *$ & $* *$ & $* *$ \\
\hline
\end{tabular}

Values with different superscript roman letters (a-e) in the same column indicate statistically significant differences at $p<0.05 ; ; n=5$; $0.001^{* * *} ; 0.01^{* *} ; 0.05^{*}$.

In the current study, it has also been demonstrated that microorganisms used for fermentation and plum cultivar affected the rate of fermentation. Generally, the highest fermentation rate was noted in Węgierka Dabrowicka and Čačanska Lepotica (except spontaneous fermentation) (Figures 1-4), however, in the case of the first cultivar, weight loss occurred successively throughout the whole fermentation process. It could be associated 
to the fact that this cultivar demonstrated the highest titratable acidity $(12.22 \mathrm{~g}$ of malic acid/L) and period of adaptation of yeast was the longest. The final weight losses were highest during fermentation carried out with both strains of S. cerevisiae S1 (about $8 \%$ for Čačanska Lepotica and 6\% for Wegierka Dabrowicka-S. cerevisiae S1, and about $6 \%$ for both cultivars fermented with Ethanol RED S. cerevisiae). However, musts obtained from those cultivars fermented spontaneously demonstrated the lowest fermentation efficiency. A possible explanation of that phenomenon is that microorganisms involved in spontaneous fermentation required more time for the adaptation to the conditions provided for the fermentation process. We noted that the turbulent stage of fermentation was delayed and started after the fourth day. This resulted in lower weight losses, especially in variants obtained from Čačanska Lepotica. On the other hand, decreasing the rate of fermentation could minimize heat release and improve the formation of different volatiles such as terpenes [17].

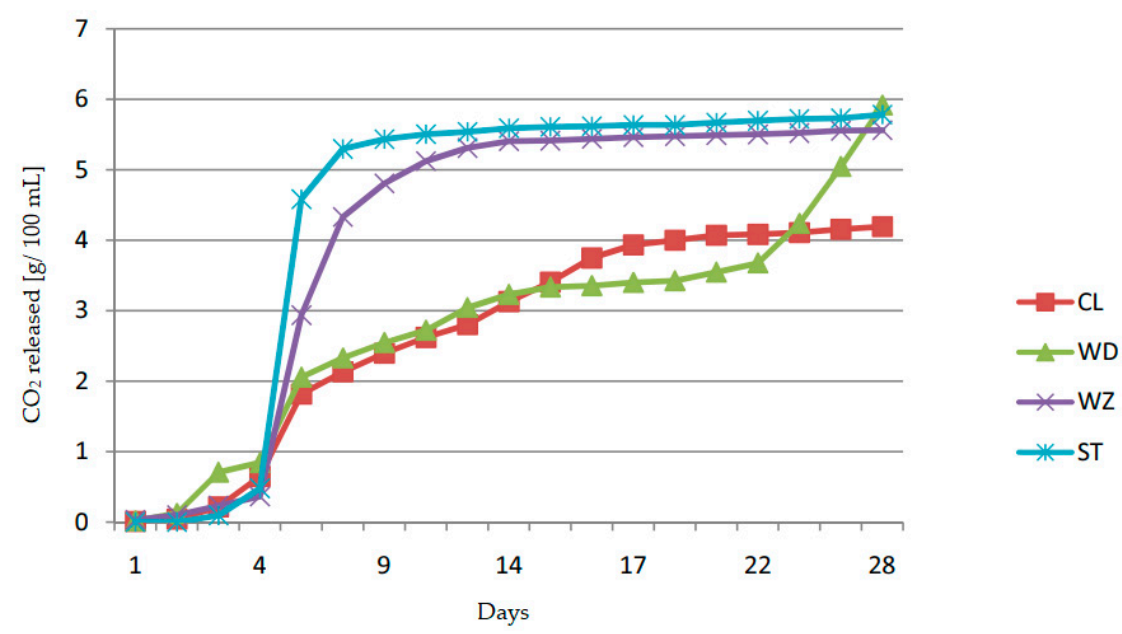

Figure 1. Fermentation dynamics of plum must fermented spontaneously, $n=5$, STD $<5 \%$. Abbreviations: CL-Čačanska Lepotica, WD—Węierka Dąbrowicka, WZ—Węgierka Zwykła, ST—Stanley.

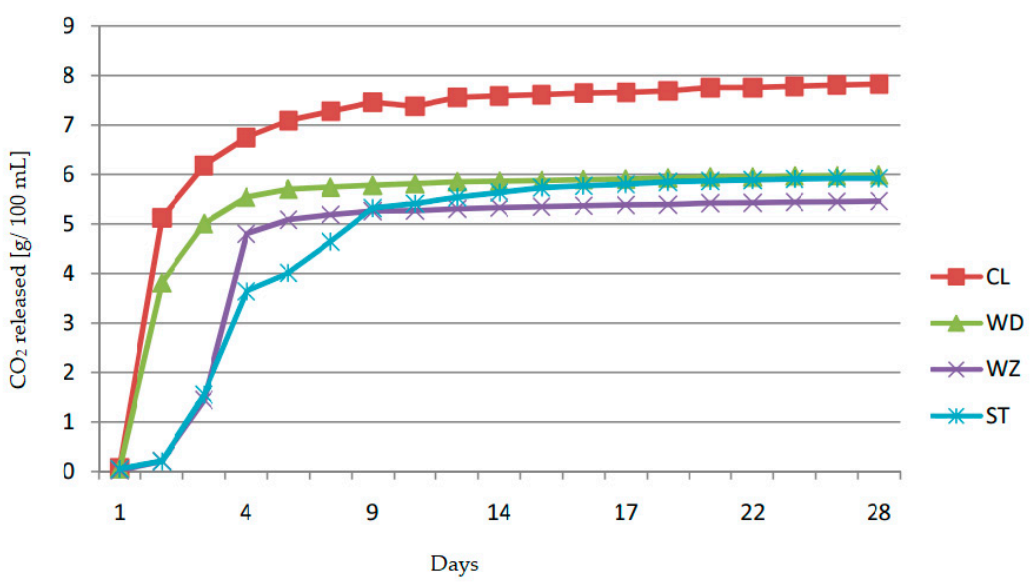

Figure 2. Fermentation dynamics of plum must fermented with $S$. cerevisiae $\mathrm{S} 1, n=5, \mathrm{STD}<5 \%$. Abbreviations: CL-Čačanska Lepotica, WD-Węgierka Dabrowicka, WZ-Węgierka Zwykła, ST-Stanley. 


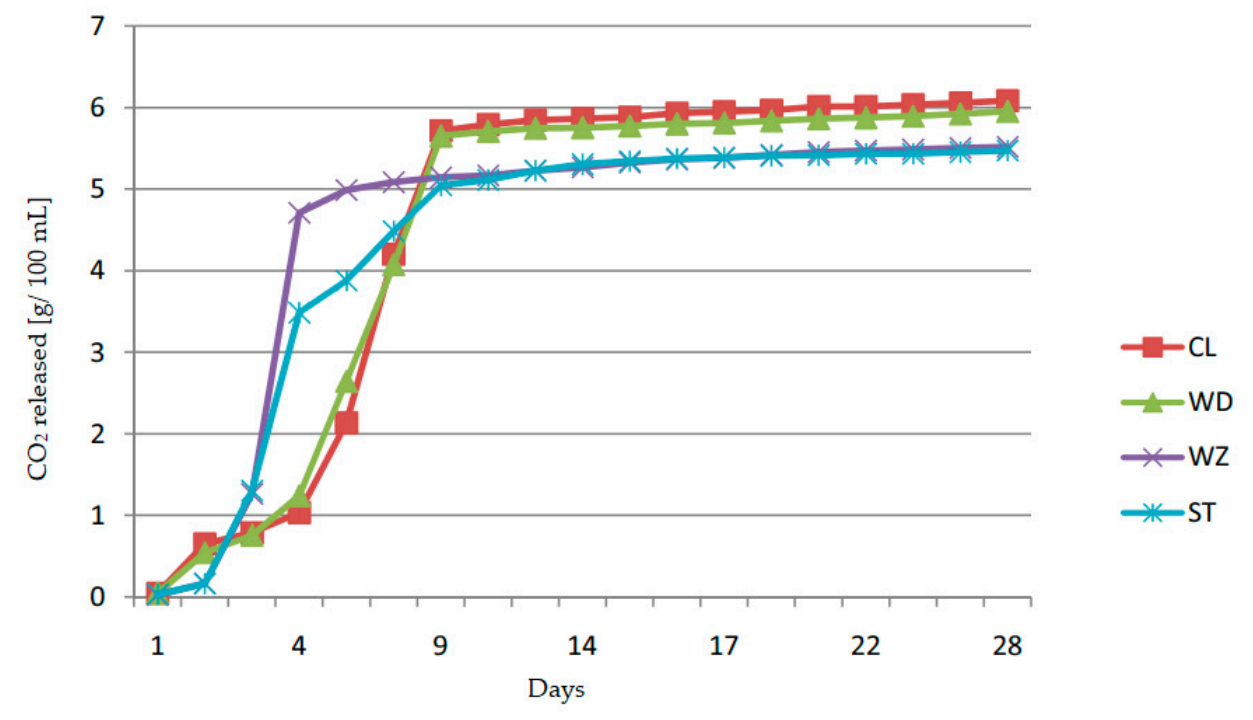

Figure 3. Fermentation dynamics of plum must fermented with $H$. uvarum $\mathrm{H} 2, n=5, \mathrm{STD}<5 \%$. Abbreviations: CL—Čačanska Lepotica, WD—Węgierka Dąbrowicka, WZ—Węgierka Zwykła, ST—Stanley.

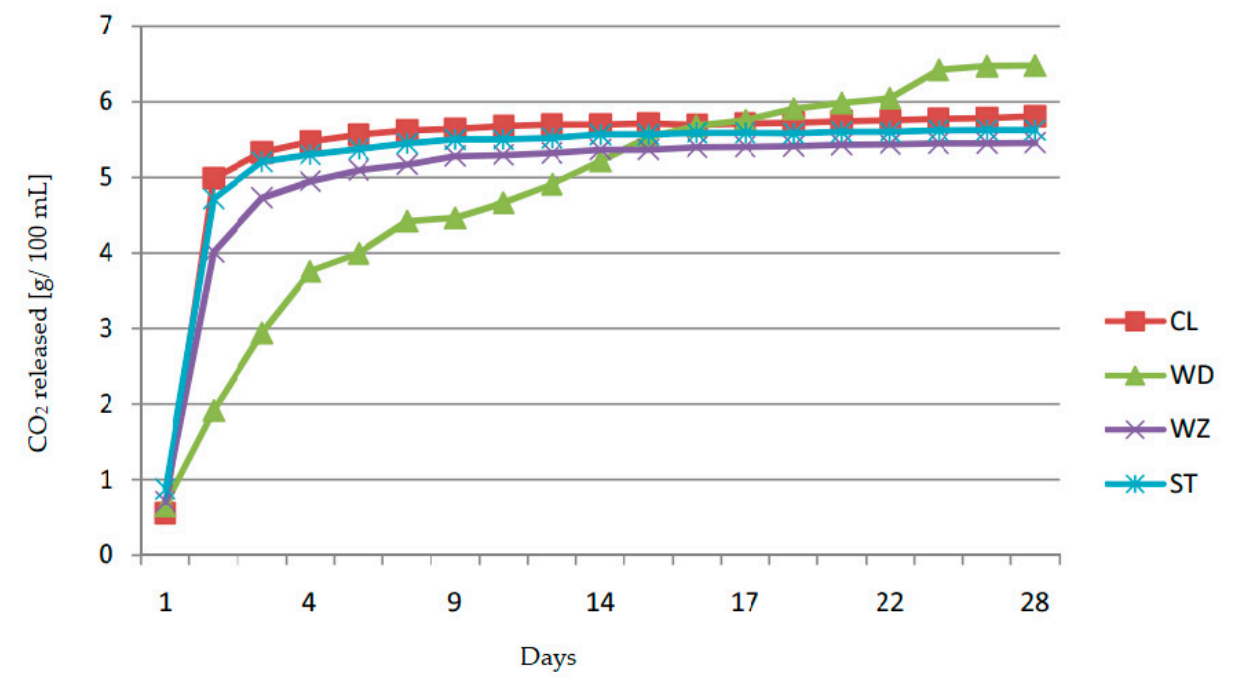

Figure 4. Fermentation dynamics of plum must fermented with Ethanol RED (S. cerevisiae), $n=5$, STD $<5 \%$. Abbreviations: CL-Čačanska Lepotica, WD-Węgierka Dąbrowicka, WZ—Węgierka Zwykła, ST—Stanley.

Analyzed plum musts had high content of sugar-free extract (26.7-41.4 g/L) which is affected by, e.g., polyols, nitrogenous compounds, non-volatile organic acids, tannins, vitamins, pigments, and mineral salts. The quantity of these compounds determines the proper growth of microorganisms during fermentation [4]. Such high level of that parameter could be connected to the high level of titratable acidity of analyzed musts, which ranged from 9.06 (Węgierka Zwykła) to $12.22 \mathrm{~g}$ of malic acid/L (Węgierka Dąbrowicka). Similar or slightly lower titratable acidity (9.76 g of malic acid/L_Węgierka Zwykła and $7.55 \mathrm{~g}$ of malic acid/L-Węgierka Dąbrowicka) was demonstrated by Satora et al., 2017 [4]. On the other hand, Pashova et al., 2006 [14] showed that Red Damson and Blackthorn cultivars demonstrated higher acidity, which were 18.1 and $27.3 \mathrm{~g}$ of malic acid/L, respectively. The prevailing organic acid in analyzed plum musts in the current study was malic acid, which was also confirmed in other published studies $[14,18]$. Quantities of citric and succinic acids were lower and in most cases did not exceed $1 \mathrm{~g} / \mathrm{L}$ (Table 3). 
Table 3. Profile of organic acids, sugars, and glycerol concentrations in plum jerkums.

\begin{tabular}{|c|c|c|c|c|c|c|c|c|c|c|}
\hline $\begin{array}{l}\text { Plum } \\
\text { Cultivar }\end{array}$ & $\begin{array}{c}\text { Type of } \\
\text { Fermentation }\end{array}$ & $\begin{array}{l}\text { Citric } \\
\text { Acid }\end{array}$ & $\begin{array}{l}\text { Malic } \\
\text { Acid }\end{array}$ & $\begin{array}{l}\text { Succinic } \\
\text { Acid }\end{array}$ & $\begin{array}{l}\text { Lactic } \\
\text { Acid }\end{array}$ & $\begin{array}{l}\text { Acetic } \\
\text { Acid }\end{array}$ & Glycerol & Fructose & Sucrose & Glucose \\
\hline \multirow{4}{*}{$\begin{array}{l}\text { Wẹgierka } \\
\text { Zwykła }\end{array}$} & S. cerevisiae S1 & $0.29^{a}$ & $0.99^{c}$ & $2.01^{\mathrm{a}}$ & $9.38^{a}$ & $0.28^{c}$ & $3.10^{d}$ & $0.47^{\mathrm{d}}$ & $1.44^{\mathrm{b}}$ & $2.79 \mathrm{bc}$ \\
\hline & H. uvarum $\mathrm{H} 2$ & $0.00^{\mathrm{d}}$ & $0.00^{\mathrm{d}}$ & $1.39^{\mathrm{b}}$ & $0.48^{\mathrm{d}}$ & $0.59 \mathrm{bc}$ & $2.54^{\mathrm{d}}$ & $1.69^{\mathrm{c}}$ & $0.42^{\mathrm{d}}$ & $0.00^{\mathrm{d}}$ \\
\hline & $\begin{array}{l}\text { Spontaneous } \\
\text { fermentation }\end{array}$ & $0.00^{\mathrm{d}}$ & $0.00^{\mathrm{d}}$ & $0.12^{d}$ & $0.75^{\mathrm{d}}$ & $0.47^{\mathrm{c}}$ & $5.42^{\mathrm{c}}$ & $1.01^{\mathrm{c}}$ & $0.16^{\mathrm{d}}$ & $0.00^{\mathrm{d}}$ \\
\hline & Ethanol RED & $0.00^{\mathrm{d}}$ & $0.00^{\mathrm{d}}$ & $0.17^{\mathrm{d}}$ & $0.89^{\mathrm{d}}$ & $0.69^{b}$ & $5.14^{c}$ & $0.22^{\mathrm{d}}$ & $0.59^{\mathrm{d}}$ & $2.16^{b}$ \\
\hline \multirow{4}{*}{$\begin{array}{l}\text { Wẹgierka } \\
\text { Dąbrowicka }\end{array}$} & S. cerevisiae S1 & $0.19^{b}$ & $3.50^{\mathrm{a}}$ & $2.15^{a}$ & $6.94^{\mathrm{b}}$ & $0.84^{\mathrm{b}}$ & $6.92^{b c}$ & $0.61^{\mathrm{d}}$ & $0.32^{\mathrm{d}}$ & $2.63^{b c}$ \\
\hline & H. uvarum $\mathrm{H} 2$ & $0.24^{\mathrm{b}}$ & $3.21^{\mathrm{a}}$ & $2.26^{a}$ & $5.39 \mathrm{bc}$ & $0.93^{a b}$ & $6.25^{b c}$ & $0.00^{\mathrm{e}}$ & $2.46^{\mathrm{a}}$ & $4.14^{\mathrm{b}}$ \\
\hline & $\begin{array}{l}\text { Spontaneous } \\
\text { fermentation }\end{array}$ & $0.34^{\mathrm{a}}$ & $1.07^{c}$ & $1.29^{b}$ & $4.62^{c}$ & $0.34^{c}$ & $5.01^{\mathrm{c}}$ & $6.58^{\mathrm{b}}$ & $1.16^{\mathrm{c}}$ & $1.45^{\mathrm{c}}$ \\
\hline & Ethanol RED & $0.29^{a}$ & $3.32^{\mathrm{a}}$ & $1.91^{\mathrm{ab}}$ & $3.19^{d}$ & $0.57^{b c}$ & $8.88^{a b}$ & $2.35^{c}$ & $0.00^{\mathrm{d}}$ & $9.85^{\mathrm{a}}$ \\
\hline \multirow{4}{*}{ Stanley } & S. cerevisiae S1 & $0.14^{\mathrm{c}}$ & $1.23^{c}$ & $1.57^{\mathrm{b}}$ & $6.38^{\mathrm{b}}$ & $0.25^{\mathrm{c}}$ & $7.93^{b}$ & $11.28^{\mathrm{a}}$ & $0.85^{\mathrm{cd}}$ & $0.65^{\mathrm{cd}}$ \\
\hline & H. uvarum $\mathrm{H} 2$ & $0.07^{\mathrm{d}}$ & $0.00^{\mathrm{d}}$ & $1.16^{\mathrm{b}}$ & $2.99^{c}$ & $1.07^{\mathrm{a}}$ & $6.65^{b c}$ & $12.66^{a}$ & $1.53^{b}$ & $0.84^{\mathrm{c}}$ \\
\hline & $\begin{array}{l}\text { Spontaneous } \\
\text { fermentation }\end{array}$ & $0.15^{c}$ & $0.10^{\mathrm{d}}$ & $0.73^{c}$ & $3.32^{c}$ & $0.67^{b}$ & $6.67^{b c}$ & $10.72^{a}$ & $2.08^{a b}$ & $0.91^{\mathrm{c}}$ \\
\hline & Ethanol RED & $0.23^{b}$ & $2.97^{\mathrm{a}}$ & $2.39^{\mathrm{a}}$ & $6.39^{b}$ & $1.29^{\mathrm{a}}$ & $10.02^{a}$ & $14.05^{\mathrm{a}}$ & $1.52^{b}$ & $0.00^{\mathrm{d}}$ \\
\hline \multirow{4}{*}{$\begin{array}{l}\text { Čačanska } \\
\text { Lepotica }\end{array}$} & S. cerevisiae S1 & $0.20^{\mathrm{b}}$ & $2.00^{\mathrm{b}}$ & $2.38^{a}$ & $8.45^{\mathrm{a}}$ & $0.25^{\mathrm{c}}$ & $5.50^{\mathrm{c}}$ & $2.50^{c}$ & $1.33^{c}$ & $0.00^{\mathrm{d}}$ \\
\hline & H. uvarum $\mathrm{H} 2$ & $0.16^{c}$ & $2.36^{\mathrm{ab}}$ & $2.58^{a}$ & $6.51^{b}$ & $0.18^{c}$ & $5.73^{c}$ & $3.32^{c}$ & $0.00^{\mathrm{d}}$ & $0.01^{\mathrm{d}}$ \\
\hline & $\begin{array}{l}\text { Spontaneous } \\
\text { fermentation }\end{array}$ & $0.28^{a}$ & $0.35^{\mathrm{d}}$ & $1.43^{\mathrm{b}}$ & $5.98^{\mathrm{b}}$ & $1.33^{\mathrm{a}}$ & $5.70^{\mathrm{c}}$ & $0.00^{\mathrm{e}}$ & $0.21^{\mathrm{d}}$ & $0.84^{\mathrm{c}}$ \\
\hline & Ethanol RED & $0.25^{\mathrm{ab}}$ & $3.56^{\mathrm{a}}$ & $2.33^{a}$ & $5.50^{\mathrm{bc}}$ & $0.36^{c}$ & $6.41^{b c}$ & $0.33^{d}$ & $0.00^{\mathrm{d}}$ & $5.91^{b}$ \\
\hline \multicolumn{2}{|c|}{ SD pooled } & 0.06 & 0.92 & 0.57 & 1.35 & 0.27 & 1.94 & 1.37 & 0.53 & 0.35 \\
\hline \multicolumn{2}{|c|}{ Significance } & $* * *$ & $* *$ & $* * *$ & $* *$ & $* * *$ & $* *$ & $* *$ & $* * *$ & $* *$ \\
\hline
\end{tabular}

Values with different superscript roman letters (a-e) in the same column indicate statistically significant differences at $p<0.05 ; n=5$; $0.001^{* * *} ; 0.01^{* *}$.

The level of titratable acidity significantly decreased after fermentation (Table 2), which was probably caused by the assimilation of some organic acids, mainly malic and citric acids, in which concentration also decreased the most significantly among detected acids. However, after fermentation, concentration of succinic acid increased, especially in samples fermented with Hanseniaspora uvarum $\mathrm{H} 2$ and Saccharomyces cerevisiae $\mathrm{S} 1$ isolated from plum fruits (Table 3). Succinic acid is a common by-product of alcoholic fermentation and it is confirmed that it is a dominant non-volatile carboxylic acid synthetized by yeasts. It must be highlighted that succinic acid plays a major role in shaping the taste of wine [19]. Succinic acid could originate from either sugar or amino acid catabolism of yeast; however, it is connected to growth conditions and available nitrogen sources. Therefore, higher concentration of that organic acid in fermented musts produced from Wegierka Dabrowicka and Čačanska Lepotica could be related to a higher concentration of free amino nitrogen in those plum cultivars. Moreover, reactions of tricarboxylic acid cycle determine its direct formation [19].

Volatile acidity of wines is mostly built up by the acetic acid. That substance could be formed in biochemical reaction during fermentation or could be directly produced by acetic or lactic acid bacteria (LAB). When acetic acid is present above a certain concentration $(1.2 \mathrm{~g} / \mathrm{L}$ volatile acidity expressed as acetic acid), it decreases the quality of wine and it also inhibits the performance of yeasts $[20,21]$. A slightly higher amount of this compound was present in samples obtained from Stanley cultivar fermented with Ethanol RED and from Čačanska Lepotica fermented spontaneously. We did not notice any correlation between concentrations of acetic and lactic acids-low concentrations of acetic acid was determined in samples that contained significant quantities of lactic acid and the other way around. 
Lactic acid was detected in all analyzed fermented plum musts and its quantities ranged from 0.48 to $9.38 \mathrm{~g} / \mathrm{L}$. Highest concentration of lactic acid was present in samples fermented with S. cerevisiae S1. It is possible that during the fermentation of those variant lactic acid bacteria (LAB) produced that acid. Based on the profile of organic acids (Table 3) it could be presumed that malolactic fermentation did not occur before the time the samples were collected because, in the majority of cases, the concentration of malic acid was higher in all variants fermented with $S$. cerevisiae S1.

Glycerol enhances taste characteristics, defines texture of a wine, and as such its bouquet, taste, and smell. On the contrary to the compounds described above, it is mostly produced by yeast [22]. Glycerol contributes to the sugar-free extract so it is not surprising that values of that parameter demonstrated in the current study were relatively high $(2.54-10.02 \mathrm{~g} / \mathrm{L})$.

The concentration of free amino nitrogenous compounds in plum musts contributes to the aroma of obtained jerkums. It ranged from $119.6 \mathrm{mg} / \mathrm{L}$ to $212.1 \mathrm{mg} / \mathrm{L}$ and it decreased after fermentation, which could be possibly due to a high demand for nitrogenous substances by microorganisms present in those musts. This group of nutrients affects the rate of fermentation by enhancing growth of microorganisms. Moreover, it could prevent the formation of stuck or the occurrence of sluggish fermentation. Those compounds could be also utilized for the formation of higher alcohols. It seems that branched chain aromatic amino acids are the most important in those processes [23].

\subsection{Volatile Compounds of Fresh Plum Musts}

Qualitative and quantitative composition of volatiles of plums is very diverse and statistically significantly varies among cultivars. More than 50 volatiles were identified in plum musts of which esters were the dominant group. Among them, ethyl acetate, ethyl butanoate, butyl butanoate, and ethyl decanoate were the most abundant (Table 4). Those esters (except butyl butanoate) were also present in fermented plum musts. Esters contribute to floral and fruity aroma notes in fruit and those esters have sweet, pineapple, banana, and nut-like aroma, respectively $[24,25]$. Some of those compounds were characteristic for particular cultivars, e.g., butyl butanoate for Wegierka Zwykła, hexyl hexanoate for Węgierka Zwykła and Stanley, ethyl tridecanoate for Wẹgierka Dąbrowicka and Čačanska Lepotica. Due to numerous transformations of volatile compounds during fermentation, we could not assign specific esters to plum jerkums obtained from particular cultivars. However, we observed that samples fermented with S. cerevisiae $\mathrm{S} 1$ or H. uvarum $\mathrm{H} 2$ demonstrated the highest concentration of esters, followed by spontaneous fermentation and musts fermented with Ethanol RED (four times lower concentration, e.g., methyl hexanoate, isoamyl lactate). Differences in the concentration of esters are associated with the fact that esters are produced during fermentation by yeast cells in an enzyme-catalyzed intracellular reaction [26]. Generally, H. uvarum is known as a good ester producer and it increases formation of some acetate esters. According to the literature, $\mathrm{H}$. uvarum were able to produce ethyl acetate, geranyl acetate, and isoamyl acetate in model solution (GPYM-glucose, peptone, yeast extract, malt extract medium) [27]. Combined cultures of Hanseniaspora spp. and S. cerevisiae increased the formation of esters and improved sensory properties of wines [28]. It is worth mentioning that in the current study, the samples fermented with S. cerevisiae S1 demonstrated the highest concentration of isoamyl lactate. This could be related to the higher concentration of lactic acid in samples fermented with this type of yeast, which probably resulted from spontaneous malolactic fermentation. 
Table 4. The composition of volatile compounds in musts obtained from various plum cultivars $[\mu \mathrm{g} / \mathrm{L}]$.

\begin{tabular}{|c|c|c|c|c|c|c|c|c|}
\hline $\begin{array}{l}\text { Compounds } \\
{[\mu \mathrm{g} / \mathrm{L}]}\end{array}$ & $\mathrm{LRI}^{2}$ & $\begin{array}{l}\text { Węgierka } \\
\text { Zwykła }\end{array}$ & $\begin{array}{c}\text { Węgierka } \\
\text { Dąbrowicka }\end{array}$ & Stanley & $\begin{array}{l}\text { Čačanska } \\
\text { Lepotica }\end{array}$ & $\mathrm{OT}^{4}$ & Sig. & $\begin{array}{c}\text { Characteristic } \\
\text { Aroma }\end{array}$ \\
\hline \multicolumn{9}{|l|}{ Esters } \\
\hline Ethyl acetate & 868 & $6667^{b}$ & $5966^{b}$ & $6540^{b}$ & $10118^{a}$ & 5000 & * & sweet, solvent \\
\hline Isobutyl acetate & 1011 & $5.5^{b}$ & $10.6^{\mathrm{ab}}$ & $5.4^{b}$ & $16.4^{\mathrm{a}}$ & 66 & ** & fig-like, banana \\
\hline Ethyl butanoate ${ }^{3}$ & 1033 & $112^{b}$ & $112^{b}$ & $119^{b}$ & $229^{a}$ & 1 & $* * *$ & pineapple \\
\hline $\begin{array}{c}\text { Ethyl } \\
\text { 2-methylbutyrate }\end{array}$ & 1048 & $7.6^{a}$ & $8.0^{\mathrm{a}}$ & $10.9^{a}$ & $12.3^{\mathrm{a}}$ & 0.3 & ns & berry, tropical \\
\hline Butyl butanoate & 1215 & $151^{\mathrm{a}}$ & $0^{b}$ & $0^{b}$ & $0^{b}$ & 100 & $* * *$ & $\begin{array}{c}\text { fruity banana } \\
\text { pineapple sweet }\end{array}$ \\
\hline Ethyl hexanoate & 1230 & $23^{a}$ & $15^{\mathrm{a}}$ & $21^{\mathrm{a}}$ & $24^{\mathrm{a}}$ & 1 & ns & $\begin{array}{l}\text { apple peel, } \\
\text { pineapple }\end{array}$ \\
\hline Hexyl butanoate & 1416 & $10.5^{\mathrm{a}}$ & $0^{b}$ & $10.7^{\mathrm{a}}$ & $2.2^{b}$ & 250 & $* * *$ & $\begin{array}{l}\text { green, fruity, estry } \\
\text { vegetative }\end{array}$ \\
\hline Ethyl octanoate & 1438 & $7.8^{\mathrm{ab}}$ & $6.7^{\mathrm{b}}$ & $9.2^{a b}$ & $12.4^{\mathrm{a}}$ & 15 & * & Fruity, winey, sweet \\
\hline $\begin{array}{l}\text { 3-hexenyl } \\
\text { butanoate }\end{array}$ & 1464 & $0.5^{\mathrm{bc}}$ & $0^{c}$ & $1.8^{\mathrm{b}}$ & $10.1^{\mathrm{a}}$ & 20,000 & $* * *$ & $\begin{array}{l}\text { Fresh, green apple, } \\
\text { fruity }\end{array}$ \\
\hline Hexyl hexanoate & 1585 & $0.6^{a}$ & $0^{b}$ & $0.6^{\mathrm{a}}$ & $0^{b}$ & 6.4 & ** & Herbaceous \\
\hline Ethyl decanoate & 1612 & $311^{b}$ & $382^{a b}$ & $311^{b}$ & $474^{\mathrm{a}}$ & 510 & * & $\begin{array}{l}\text { Sweet, fatty, nut-like, } \\
\text { winey }\end{array}$ \\
\hline $\begin{array}{l}\text { 1-methylethyl } \\
\text { dodecanoate }\end{array}$ & 1800 & $2.4^{\mathrm{d}}$ & $24.6^{\mathrm{a}}$ & $9.3^{b}$ & $5.3^{c}$ & - & $* * *$ & - \\
\hline Ethyl dodecanoate & 1812 & $210^{a}$ & $261^{a}$ & $242^{\mathrm{a}}$ & $208^{a}$ & 2000 & ns & Oily, fatty, floral \\
\hline Ethyl tridecanoate & 1994 & $0^{b}$ & $34^{a}$ & $0^{b}$ & $60^{a}$ & - & $* * *$ & - \\
\hline $\begin{array}{c}\text { Ethyl } \\
\text { tetradecanoate }\end{array}$ & 2093 & $37^{b}$ & $44^{b}$ & $35^{b}$ & $85^{\mathrm{a}}$ & 4000 & $* * *$ & Mild, waxy, soapy \\
\hline \multicolumn{9}{|l|}{ Alcohols } \\
\hline 1-butanol & 1144 & $18.6^{b}$ & $0^{b}$ & $83.7^{\mathrm{a}}$ & $0^{b}$ & 500 & $* * *$ & $\begin{array}{l}\text { banana harsh } \\
\text { alcoholic sweet }\end{array}$ \\
\hline 1-hexanol & 1348 & $2088^{b c}$ & $3001^{b}$ & $8691^{a}$ & $1112^{c}$ & 2500 & $* * *$ & $\begin{array}{l}\text { herbal ethereal } \\
\text { alcoholic green }\end{array}$ \\
\hline 3-hexen-1-ol & 1385 & $6.0^{b}$ & $8.9^{b}$ & $4.5^{b}$ & $23.5^{a}$ & 70 & $* * *$ & $\begin{array}{l}\text { grassy-green freshly } \\
\text { cut grass }\end{array}$ \\
\hline 2-hexen-1-ol & 1409 & $10.6^{\mathrm{a}}$ & $4.9^{b}$ & $4.6^{\mathrm{b}}$ & $2.2^{b}$ & 400 & $* * *$ & Sharp green leafy \\
\hline 2-ethyl-1-hexanol & 1487 & $2.8^{\mathrm{a}}$ & $0^{c}$ & $2.2^{\mathrm{ab}}$ & $1.5^{\mathrm{b}}$ & 138 & $* * *$ & $\begin{array}{c}\text { citrus fresh floral } \\
\text { oily sweet }\end{array}$ \\
\hline 1-nonanol & 1642 & $10.2^{a}$ & $18.4^{\mathrm{a}}$ & $12.0^{\mathrm{a}}$ & $10.3^{a}$ & 50 & ns & citrus \\
\hline Benzyl alcohol & 1858 & $4.7^{\mathrm{a}}$ & $2.5^{\mathrm{a}}$ & $3.7^{\mathrm{a}}$ & $4.0^{\mathrm{a}}$ & 10,000 & ns & $\begin{array}{c}\text { floral rose phenolic } \\
\text { balsamic }\end{array}$ \\
\hline Phenol & 1972 & $0.1^{b}$ & $0.5^{\mathrm{ab}}$ & $0.5^{\mathrm{ab}}$ & $0.9^{a}$ & 5900 & * & $\begin{array}{c}\text { phenolic plastic } \\
\text { rubber }\end{array}$ \\
\hline 2-phenoxyethanol & 2114 & $0.5^{\mathrm{a}}$ & $0^{b}$ & $0.6^{\mathrm{a}}$ & $0.4^{\mathrm{ab}}$ & - & * & $\begin{array}{l}\text { mild rose balsam } \\
\text { cinnamyl }\end{array}$ \\
\hline
\end{tabular}


Table 4. Cont.

\begin{tabular}{|c|c|c|c|c|c|c|c|c|}
\hline $\begin{array}{l}\text { Compounds } \\
{[\mu \mathrm{g} / \mathrm{L}]}\end{array}$ & LRI $^{2}$ & $\begin{array}{l}\text { Węgierka } \\
\text { Zwykła }\end{array}$ & $\begin{array}{c}\text { Węgierka } \\
\text { Dąbrowicka }\end{array}$ & Stanley & $\begin{array}{c}\text { Čačanska } \\
\text { Lepotica }\end{array}$ & $\mathrm{OT}^{4}$ & Sig. & $\begin{array}{c}\text { Characteristic } \\
\text { Aroma }\end{array}$ \\
\hline \multicolumn{9}{|l|}{$\begin{array}{l}\text { Carbonyl } \\
\text { compounds }\end{array}$} \\
\hline $\begin{array}{l}\text { 5-methyl-3- } \\
\text { hexanone }\end{array}$ & 1069 & $2205^{b}$ & $3140^{\mathrm{a}}$ & $1753^{b}$ & $3365^{a}$ & - & $* * *$ & pleasant fruity \\
\hline Hexanal & 1076 & $1122^{b}$ & $7783^{a}$ & $7118^{a}$ & $8562^{a}$ & $4.5-5$ & $* * *$ & grassy \\
\hline $\begin{array}{l}\text { 4-methyl-2- } \\
\text { hexanone }\end{array}$ & 1113 & $0^{\mathrm{b}}$ & $1.5^{\mathrm{a}}$ & $2.8^{a}$ & $2.4^{\mathrm{a}}$ & - & $* * *$ & pleasant fruity \\
\hline 2-hexenal & 1199 & $291^{b c}$ & $467^{\mathrm{ab}}$ & $549^{a}$ & $134^{c}$ & 17 & $* *$ & green \\
\hline Nonanal $^{3}$ & 1392 & $4.0^{\mathrm{ab}}$ & $2.5^{b}$ & $5.9^{a}$ & $2.3^{b}$ & 1 & $* *$ & $\begin{array}{c}\text { aldehydic rose } \\
\text { orange peel }\end{array}$ \\
\hline 2,4-hexadienal & 1406 & $0.3^{\mathrm{a}}$ & $0.6^{\mathrm{a}}$ & $0.8^{\mathrm{a}}$ & $0.6^{\mathrm{a}}$ & 60 & ns & $\begin{array}{l}\text { Green, fruity, } \\
\text { aldehydic, citrus }\end{array}$ \\
\hline Decanal & 1491 & $7.7^{\mathrm{a}}$ & $0^{b}$ & $2.0^{\mathrm{b}}$ & $0^{b}$ & 2 & $* *$ & $\begin{array}{l}\text { aldehydic orange } \\
\text { peel citrus }\end{array}$ \\
\hline Benzaldehyde & 1513 & $2.1^{b}$ & $0.7^{\mathrm{b}}$ & $2.2^{b}$ & $6.1^{a}$ & 350 & $* *$ & spicy bitter-almond \\
\hline Acetophenone & 1640 & $10.8^{a}$ & $4.0^{\mathrm{bc}}$ & $0^{c}$ & $7.7^{\mathrm{ab}}$ & 65 & $* * *$ & $\begin{array}{c}\text { pungent hawthorn } \\
\text { almond }\end{array}$ \\
\hline \multicolumn{9}{|l|}{ Terpenoids } \\
\hline p-Cymene & 1259 & $1.2^{b c}$ & $0.9^{c}$ & $1.6^{\mathrm{b}}$ & $2.6^{a}$ & - & $* * *$ & $\begin{array}{l}\text { solvent, gasoline, } \\
\text { citrus }\end{array}$ \\
\hline Bornylene & 1506 & $0.7^{\mathrm{a}}$ & $0^{b}$ & $0^{b}$ & $0.3^{a b}$ & - & $* *$ & - \\
\hline Linalool & 1536 & $13.7^{\mathrm{a}}$ & $1.4^{\mathrm{c}}$ & $4.3^{b}$ & $5.0^{\mathrm{b}}$ & 6 & $* * *$ & $\begin{array}{l}\text { Floral, woody, } \\
\text { lavender }\end{array}$ \\
\hline Calamenene & 1567 & $0.5^{\mathrm{b}}$ & $3.2^{a}$ & $1.3^{a b}$ & $0.5^{b}$ & - & $* * *$ & Herb spice \\
\hline B-Cyclocitral & 1595 & $0.5^{b}$ & $0^{c}$ & $0^{\mathrm{c}}$ & $0.9^{a}$ & 5 & $* * *$ & Minty, citrus \\
\hline Menthol & 1617 & $0.8^{\mathrm{a}}$ & $0.2^{b}$ & $0^{b}$ & $0.2^{a b}$ & - & * & $\begin{array}{l}\text { Cooling, fresh, } \\
\text { sweet, minty }\end{array}$ \\
\hline Damascenone & 1804 & $3.6^{b}$ & $17.9^{a}$ & $6.8^{\mathrm{b}}$ & $4.0^{\mathrm{b}}$ & 0.002 & $* *$ & Apple, rose honeys \\
\hline Geraniol & 1816 & $0.3^{\mathrm{a}}$ & $1.4^{\mathrm{a}}$ & $1.4^{\mathrm{a}}$ & $1.4^{\mathrm{a}}$ & 40 & ns & $\begin{array}{l}\text { floral fruity rose } \\
\text { waxy citrus }\end{array}$ \\
\hline Geranyl acetone & 1828 & $3.3^{a}$ & $1.3^{\mathrm{a}}$ & $1.4^{\mathrm{a}}$ & $3.1^{\mathrm{a}}$ & 60 & ns & $\begin{array}{c}\text { rose leaf magnolia } \\
\text { aldehydic }\end{array}$ \\
\hline B-ionone & 1918 & $0.6^{b}$ & $0.9^{a b}$ & $2.2^{\mathrm{a}}$ & $2.1^{a}$ & 7 & * & $\begin{array}{l}\text { violet raspberry } \\
\text { woody fruity }\end{array}$ \\
\hline p-cresol & 1967 & $0.3^{\mathrm{a}}$ & $0.2^{\mathrm{a}}$ & $0.2^{\mathrm{a}}$ & $0.1^{\mathrm{a}}$ & 55 & ns & phenolic narcissus \\
\hline Eugenol & 2136 & $0.4^{\mathrm{a}}$ & $1.6^{\mathrm{a}}$ & $1.0^{\mathrm{a}}$ & $1.4^{\mathrm{a}}$ & 6 & ns & $\begin{array}{l}\text { sweet spicy clove } \\
\text { woody }\end{array}$ \\
\hline \multicolumn{9}{|l|}{ Lactones } \\
\hline$\Gamma$-nonanolactone & 2128 & $1.1^{\mathrm{ab}}$ & $2.0^{\mathrm{a}}$ & $1.2^{\mathrm{ab}}$ & $0.5^{b}$ & 65 & * & $\begin{array}{l}\text { coconut creamy } \\
\text { waxy buttery waxy } \\
\text { peach coconut } \\
\text { buttery }\end{array}$ \\
\hline$\Gamma$-decanolactone & 2328 & $3.2^{\mathrm{a}}$ & $2.9^{\mathrm{a}}$ & $2.6^{\mathrm{a}}$ & $0.2^{b}$ & 11 & $* *$ & \\
\hline
\end{tabular}


Table 4. Cont.

\begin{tabular}{|c|c|c|c|c|c|c|c|c|}
\hline $\begin{array}{l}\text { Compounds } \\
{[\mu \mathrm{g} / \mathrm{L}]}\end{array}$ & LRI $^{2}$ & $\begin{array}{l}\text { Węgierka } \\
\text { Zwykła }\end{array}$ & $\begin{array}{c}\text { Węgierka } \\
\text { Dąbrowicka }\end{array}$ & Stanley & $\begin{array}{l}\text { Čačanska } \\
\text { Lepotica }\end{array}$ & $\mathrm{OT}^{4}$ & Sig. & $\begin{array}{c}\text { Characteristic } \\
\text { Aroma }\end{array}$ \\
\hline \multicolumn{9}{|l|}{ Hydrocarbons } \\
\hline Acenaphthene & 2154 & $1.5^{\mathrm{a}}$ & $0.8^{\mathrm{a}}$ & $1.5^{\mathrm{a}}$ & $1.4^{\mathrm{a}}$ & 80 & * & Pungent \\
\hline \multicolumn{9}{|l|}{ Other compounds } \\
\hline Dimethyl sulfoxide & 1204 & $2.0^{\mathrm{a}}$ & $2.3^{\mathrm{a}}$ & $4.3^{\mathrm{a}}$ & $2.6^{\mathrm{a}}$ & - & ns & Garlic-like \\
\hline Benzothiazole ${ }^{3}$ & 1952 & $8.8^{\mathrm{a}}$ & $7.8^{\mathrm{a}}$ & $11.0^{\mathrm{a}}$ & $9.3^{\mathrm{a}}$ & 80 & ns & $\begin{array}{c}\text { Sulphurous, rubbery, } \\
\text { burnt }\end{array}$ \\
\hline
\end{tabular}

Values with different superscript roman letters (a-e) in the same row indicate statistically significant differences at $p<0.05 ; n=5$; ns-not significant; $0.001^{* * *} ; 0.01 * * ; 0.05 *$. ${ }^{2}$ LRI-linear retention index. ${ }^{3}$ Determined semi-quantitatively by measuring the relative peak area of each identified compound, according to the NIST database, in relation to that of the internal standard. ${ }^{4}$ OT-Odor thresholds in spirits or wines [25].

The next largest groups of volatile compounds were alcohols and carbonyl compounds. Hexanol (with herbal ethereal alcoholic green aroma) was the most abundant of the nine alcohols detected in fresh musts and constituted of over $90 \%$ of alcohols. This is in agreement with the results presented in other studies which indicated hexanol as a characteristic compound in plums $[24,25,29]$. The origin of $\mathrm{C} 6$ alcohols, e.g., 1-hexanol, is related to the lipoxygenase activity, which occurs in plants mainly in fruits. It brakes unsaturated fatty acids and the products of such reaction are precursors of short chain alcohols. After fermentation, in analyzed samples some of other alcohols have occurred, e.g., propanol and isobutanol. Those compounds could be produced by yeast from amino acids and they naturally occur in wines $[30,31]$. As well as in the case of esters, the concentration of most of these compounds were higher in juices fermented with $H$. uvarum $\mathrm{H} 2$ or S. cerevisiae S1, especially in Węgierka Zwykła and Stanley cultivars in comparison to samples fermented with Ethanol RED (Table 5). Musts fermented with S. cerevisiae S1 demonstrated higher concentrations of propanol, isobutanol, butanol, and 2-phenylethanol. Concentration of the last of these compounds was also high in samples fermented with Ethanol RED (S. cerevisiae). It is claimed, that Saccharomyces genus is able to produce significant amounts of 2-phenylethanol and it is currently receiving attention as yeast having a great prospect in development of biotechnological production of this substance [32].

Increased production of higher alcohols by $H$. uvarum was claimed, but there is no information on the increased synthesis of methanol by this strain. However, according to our studies, concentration of methanol in samples fermented with $H$. uvarum $\mathrm{H} 2$ was the highest and exceeded $950 \mathrm{mg} / \mathrm{L}$. According to results presented by other authors, concentration of methanol in plum wines varies from $175 \mathrm{mg} / \mathrm{L}$ to almost $1000 \mathrm{mg} / \mathrm{L}[33,34]$. Methanol is produced by the hydrolysis of methyl ester groups in pectins from fruits, so it is present in wines and spirits. The enzyme which catalyzes that reaction (pectin methyl esterase) occurs not only in plants, but it may also be produced by various microorganisms [9]. The main reason standing behind high methanol concentrations in our samples was the application of pectinolytic preparation and using whole plum fruits, including skins and stones. 
Table 5. Aroma composition of plum jerkums [ $\mu \mathrm{g} / \mathrm{L}]$.

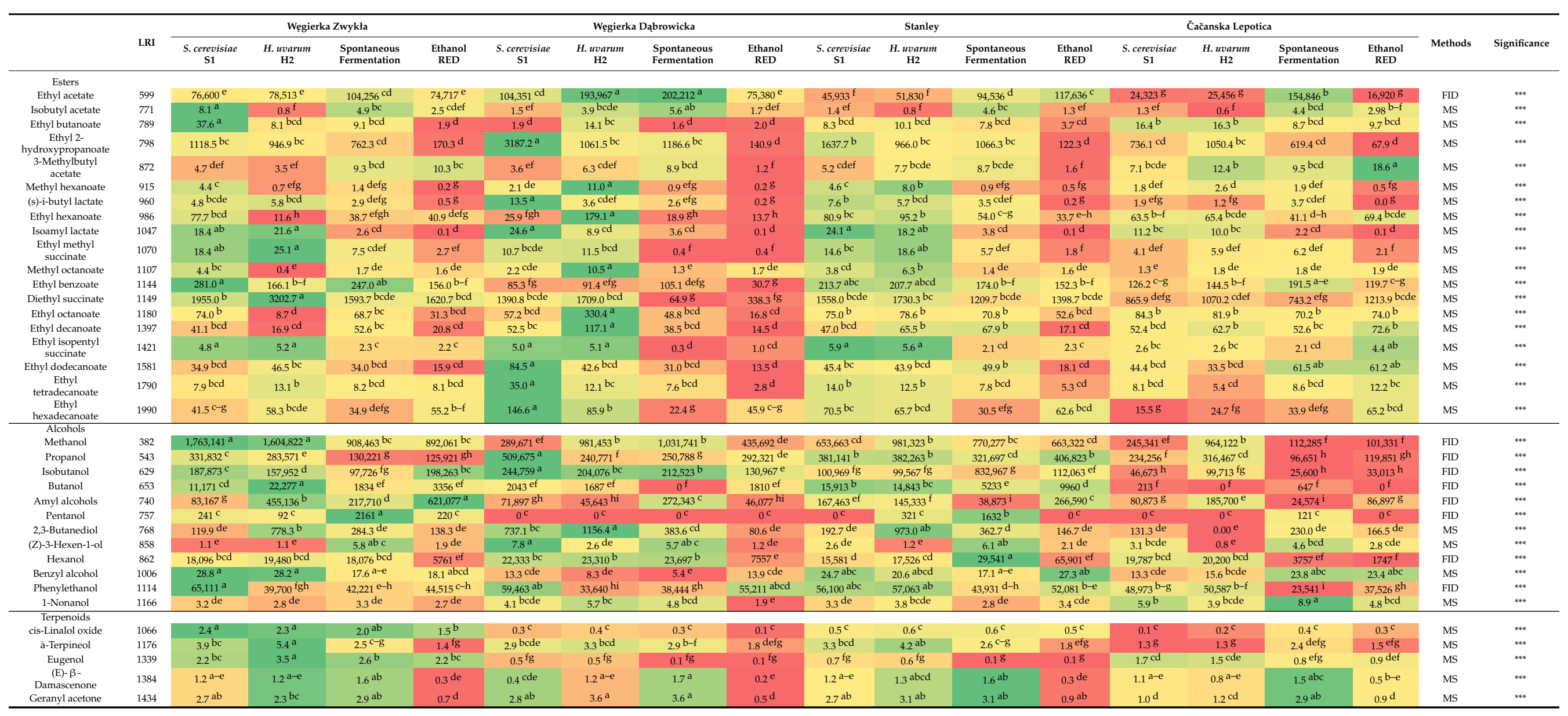


Table 5. Cont.

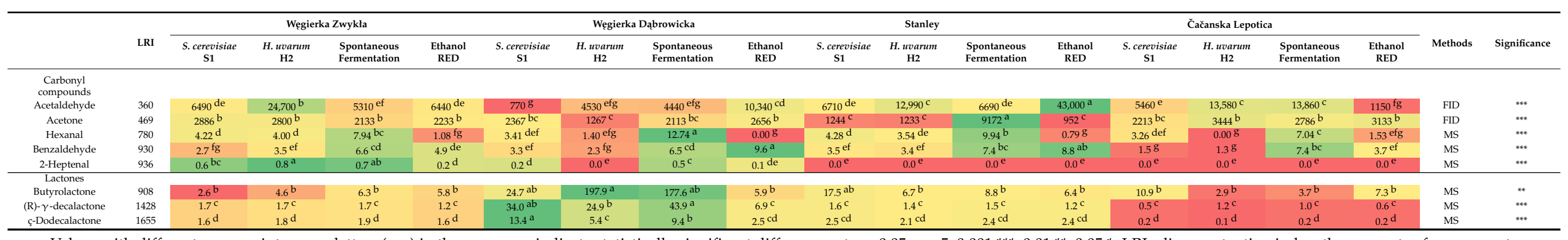

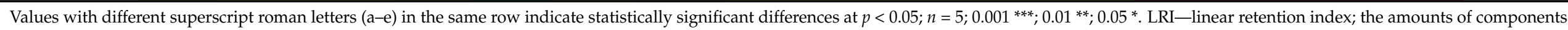

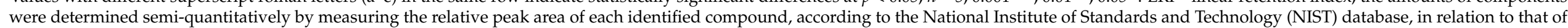

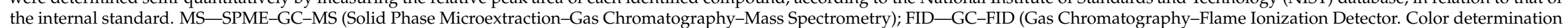

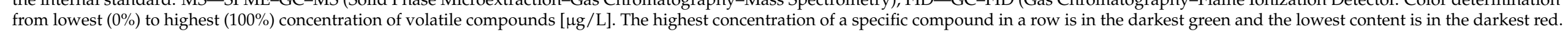


In the current study, the most abundant aldehydes in fresh musts were 5-methyl-3hexanone (fruity aroma) and hexanal (grassy scents). Moreover, except for hexanal, we identified other aldehydes and alcohols of six-carbon atoms in all cultivars. 2-hexenal, hexanal, 2-hexenol, 3-hexenol, and hexanol are characteristic for plums and contribute to the green note of the fruit. Those compounds might be formed when the fruit tissues are crushed or blended and the compounds of cytosol and cell walls are exposed to lipoxygenases. The presence of these compounds is probably due to lipoxygenase activity, which is initialized by the disruption of the fruit tissues when it is crushed or blended [35]. Other carbonyl compound identified in plum musts was nonanal (woody-like aroma) which could be found in waxes that cover the skin of plum fruit [35]. Some carbonyl compounds were characteristic for particular cultivars (regardless of the type of fermentation carried out), e.g., 2-heptenal in fermented musts obtained from Wẹgierka Dabrowicka and Węgierka Zwykład and 2-buten-1-one, from Węgierka Dabrowicka and Čačanska Lepotica.

Some of the carbonyl compounds were also identified in jerkums and their concentration was usually higher in samples fermented spontaneously than in other types of fermentation. These compounds included, e.g., hexanal, benzaldehyde, and 2-heptenal.

The acetaldehyde is one of the most important carbonyl compounds produced during fermentation, which at low levels contributes to fruity flavors, while high concentrations $(200 \mathrm{mg} / \mathrm{L})$ cause flatness in wines [36]. It was present in all analyzed jerkums. Its concentration did not exceed this value and was not dependent on the type of fermentation used.

Among terpenoids, linalool, p-cymene, geraniol, and geranyl acetone were dominant compounds in analyzed plum musts. Those compounds (with the exception of geraniol) were also detected in plums Prunus domestica L. cv. Horvin as described by Pino et al. [25]. The terpenoids, namely the monoterpenols, were reported as volatile components of fruits responsible for a wide spectrum of aromas, mostly perceived as very pleasant. The compounds are responsible for the varietal aroma of fruits, and at least some of them are present in their glycosidic compounds. The concentration of linalool, which is also present in fresh plum, may increase during technological processing, mostly, by its release from glycosidic forms. That process may occur due to the heat release [37,38].

Bornylene is an interesting compound which was not detected in plum fruit, so far, however its occurrence was confirmed in kiwi fruit and wines obtained from that fruit [39]. However, this compound was present in Węgierka Zwykła and Čačanska Lepotica cultivars in the current study. After fermentation, the profile of terpenoids was changed and the highest concentration of those compounds was determined in samples fermented spontaneously or with $H$. uvarum $\mathrm{H} 2$. Despite the fact that analyzed samples were immediately frozen after fermentation and stored at $-18{ }^{\circ} \mathrm{C}$, some volatile compounds could be oxidized or transformed into each other. Probably, linalool oxidized to cislinalool oxide (which was present after fermentation) and the aroma of the latter volatile is less intense. In addition to the oxidation processes, other enzymatic and non-enzymatic reactions occur during storage. Terpenoids are reduced by $50-60 \%$ after 3 months of storage of wines [40]. It was also noted that during that period those compounds undergo various transformations which result in the formation of cyclic compounds, ketones, lactones ( $\alpha$-(alpha), $\beta$-ionone (beta), and vitispirane [40]. Transformation of terpenoids requires appropriate conditions, e.g., in acidic solutions, geraniol is converted to the cyclic terpene $\alpha$-terpineol [41], which also occurred in jerkums (Table 5).

The last group of volatile compounds in the analyzed plums was lactones. Among them, $\gamma$-nonanolactone and $\gamma$-decanolactone were detected. Lactones are formed from the corresponding hydroxy acids. These compounds, particularly $\gamma$-lactones, are important compounds in terms of their contribution to the aroma and, in general, pleasant fruity aroma descriptors [37]. Some studies confirmed $\gamma$-dodecalactone as the major lactone in plums, especially in Japanese plum (P. salicina) and candied plum (P. domestica) $[25,37]$. Completely different lactones were found in the fermented samples- $\gamma$-butyrolactone and ç-dodecalactone. The $\gamma$-butyrolactone, the most common and important lactone in 
fermented foods, has a creamy, oily, fatty, or caramel aroma and a milky, creamy taste, with fruity peach-like after-scents [42].

\section{Conclusions}

Our research proved that fermentation type had a significant impact on chemical composition and volatile profile of plum jerkums. Among four analyzed cultivars of plums, Wegierka Zwykła and Stanley demonstrated most diverse profile of volatile compounds. Musts fermented with $H$. uvarum $\mathrm{H} 2$ presented higher concentration of terpenoids, e.g., $\alpha$-terpineol, eugenol, and esters, e.g., ethyl propanoate, methyl hexanoate, isoamyl lactate, than samples fermented with Ethanol RED. As well as in the case of esters, the concentration of some higher alcohols was higher in musts fermented with H. uvarum $\mathrm{H} 2$ or S. cerevisiae S1, e.g., propanol, isobutanol, butanol, and 2-phenylethanol. Concentration of the last of those compounds was also high in samples fermented with Ethanol RED (S. cerevisiae), which confirms that the Sacharomyces genus is able to produce significant amounts of 2-phenylethanol. Despite the fact that the majority of non-Saccharomyces yeasts produce high levels of volatile compounds, their application could cause technological disadvantages. However, in the current paper, the application of indigenous strains of yeast demonstrated desired results, e.g., highest fermentation efficiency and concentration of ethanol was determined in musts fermented with Ethanol RED (S. cerevisiae) and also with S. cerevisiae S1, while lowest efficiency was shown for musts fermented spontaneously. Based on our results it could be concluded that indigenous strains of yeast present in plums demonstrate great potential for the production of plum jerkums of high quality. In the future we are planning to involve olfactometer detector for the determination of sensory active aroma compounds.

Author Contributions: Conceptualization, P.S. and M.J.; methodology, P.S. and M.J.; software, P.S.; validation, P.S. and M.J.; formal analysis, P.S. and M.J.; investigation, P.S. and M.J.; resources, P.S. and M.J.; data curation, P.S. and M.J.; writing—original draft preparation, P.S. and M.J.; writingreview and editing, M.J.; visualization, M.J.; supervision, P.S.; project administration, P.S.; funding acquisition, P.S. All authors have read and agreed to the published version of the manuscript.

Funding: The research was financed in part by the Ministry of Science and Higher Education of Poland in the years 2012-2014 as scientific project IP2011 048371 and through a research subsidy of Department of Fermentation Technology and Microbiology, University of Agriculture in Krakow.

Institutional Review Board Statement: Not applicable.

Informed Consent Statement: Not applicable.

Data Availability Statement: Not applicable.

Conflicts of Interest: The authors declare no conflict of interest. The funders had no role in the design of the study; in the collection, analyses, or interpretation of data; in the writing of the manuscript, or in the decision to publish the results.

\section{References}

1. Januszek, M.; Satora, P.; Tarko, T. Oenological Characteristics of Fermented Apple Musts and Volatile Profile of Brandies Obtained from Different Apple Cultivars. Biomolecules 2020, 10, 853. [CrossRef] [PubMed]

2. Pischl, J. Destylaty Alkoholowe. Wytwarzanie, Teoria i Praktyka; Borus \& InterPolonus: Warszawa, Poland, 2010.

3. López, F.; Rodríguez-Bencomo, J.J.; Orriols, I.; Pérez-Correa, J.R. Science and Technology of Fruit Wine Production; Academic Press: Cambridge, MA, USA, 2017; ISBN 9780128010341.

4. Satora, P.; Kostrz, M.; Sroka, P.; Tarko, T. Chemical profile of spirits obtained by spontaneous fermentation of different varieties of plum fruits. Eur. Food Res. Technol. 2017, 243, 489-499. [CrossRef]

5. Joshi, V.K.; Panesar, P.S.; Rana, V.S.; Kaur, S. Science and Technology of Fruit Wines: An Overview; Elsevier Inc.: Amsterdam, The Netherlands, 2017; ISBN 9780128010341.

6. Januszek, M.; Satora, P.; Łukasz, W.; Tarko, T. Saccharomyces bayanus Enhances Volatile Profile of Apple Brandies. Molecules 2020, 25, 3127. [CrossRef] 
7. Du Plessis, H.; Du Toit, M.; Nieuwoudt, H.; Van der Rijst, M.; Hoff, J.; Jolly, N. Modulation of wine flavor using Hanseniaspora uvarum in combination with different Saccharomyces cerevisiae, lactic acid bacteria strains and malolactic fermentation strategies. Fermentation 2019, 5, 64. [CrossRef]

8. Walkowiak-Tomczak, D. Wpływ stopnia dojrzałości na parametry fizyczno-chemiczne i zawartość związków polifenolowych w wybranych odmianach śliwek (Prunus domestica). Nauk. Przyr. Technol. 2009, 3, 149.

9. Miljić, U.; Puškaš, V. Suitability of chosen plum cultivars (Prunus domestica L.) for fruit wine production. J. Process. Energy Agric. 2015, 19, 95-97.

10. Skotniczny, M.; Satora, P.; Panczyszyn, K.; Cioch-Skoneczny, M. Growth dynamics and diversity of yeasts during spontaneous plum mash fermentation of different varieties. Foods 2020, 9, 1054. [CrossRef] [PubMed]

11. OIV. International Organisation of Vine and Wine (OIV). Compendium of International Methods of Wine and Must Analysis; OIV: Paris, France, 2016.

12. Abernathy, D.; Spedding, G.; Starcher, B. Analysis of protein and total usable nitrogen in beer and wine using a microwell ninhydrin assay. J. Inst. Brew. 2009, 115, 122-127. [CrossRef]

13. Antalick, G.; Perello, M.-C.; de Revel, G. Development, validation and application of a specific method for the quantitative determination of wine esters by headspace-solid-phase microextraction-gas chromatography-mass spectrometry. Food Chem. 2010, 121, 1236-1245. [CrossRef]

14. Pashova, S. Chemical composition of plum fruits. J. Mt. Agric. Balk. 2006, 9, 239-249.

15. Sudar, R.; Jurković, Z.; Drugalić, K.; Tomać, I.; Jurković, V.; Viljevać, M. Sorbitol and sugar composition of plum fruit during ripening. In Proceedings of the 46th Croatian and 6th International Symposium on Agriculture, Opatija, Croatia, 14-18 February 2011.

16. France Ethanol Red ${ }^{\circledR}$ Dry Alcohol Yeast; Lesaffre Advanced Fermentation Technologies; 2010. Available online: https://www. scribd.com/document/325756425/ER-EN (accessed on 5 April 2019).

17. Tarko, T.; Kostrz, M.; Duda-Chodak, A.; Semik-Szczurak, D.; Sroka, P.; Senczyszyn, T. The effect of apple cultivars and yeast strains on selected quality parameters and antioxidant activity of fermented apple beverages. CYTA J. Food 2018, 16, 892-900. [CrossRef]

18. Usenik, V.; Marn, M.V. Sugars and organic acids in Plum fruit affected by Plum pox virus. J. Sci. Food Agric. 2016, 97, 2154-2158. [CrossRef] [PubMed]

19. Coulter, A.; Godden, P.; Pretorius, I. Succinic acid-How is it formed, what is its effect on titratable acidity, and what factors influence its concentration in wine? WINE Ind. J. 2004, 19, 16-25.

20. Vilela-Moura, A.; Schuller, D.; Mendes-Faia, A.; Silva, R.D.; Chaves, S.R.; Sousa, M.J.; Côrte-Real, M. The impact of acetate metabolism on yeast fermentative performance and wine quality: Reduction of volatile acidity of grape musts and wines. Appl. Microbiol. Biotechnol. 2011, 89, 271-280. [CrossRef] [PubMed]

21. Inês, A.; Falco, V. Lactic Acid Bacteria Contribution to Wine Quality and Safety; Intech Open: London, UK, 2018 ; pp. 53-71.

22. Valles, B.S.; Bedriñana, R.P.; Tascón, N.F.; Simón, A.Q.; Madrera, R.R. Yeast species associated with the spontaneous fermentation of cider Bele. Food Microbiol. 2007, 24, 25-31. [CrossRef]

23. Mendes-Ferreira, A.; Barbosa, C.; Lage, P.; Mendes-Faia, A. The impact of nitrogen on yeast fermentation and wine quality. Ciênc. Téc. Vitivinic. 2011, 26, 17-32.

24. Burdock, G.A. Fenaroli's Handbook of Flavor Ingredients, 4th ed.; CRC Press: Boca Raton, FL, USA, 2005; ISBN 9781439863275.

25. Pino, J.A.; Quijano, C.E. Study of the volatile compounds from plum (Prunus domestica L. cv. Horvin) and estimation of their contribution to the fruit aroma. Food Sci. Technol. 2012, 32, 76-83. [CrossRef]

26. Verstrepen, K.J.; Van Laere, S.D.M.; Vanderhaegen, B.M.P.; Derdelinckx, G.; Dufour, J.P.; Pretorius, I.S.; Winderickx, J.; Thevelein, J.M.; Delvaux, F.R. Expression levels of the yeast alcohol acetyltransferase genes ATF1, Lg-ATF1, and ATF2 control the formation of a broad range of volatile esters. Appl. Environ. Microbiol. 2003, 69, 5228-5237. [CrossRef]

27. Rojas, V.; Gil, J.V.; Piñaga, F.; Manzanares, P. Studies on acetate ester production by non-Saccharomyces wine yeasts. Int. J. Food Microbiol. 2001, 70, 283-289. [CrossRef]

28. Tristezza, M.; Tufariello, M.; Capozzi, V.; Spano, G.; Mita, G.; Grieco, F. The oenological potential of hanseniaspora uvarum in simultaneous and sequential co-fermentation with Saccharomyces cerevisiae for industrial wine production. Front. Microbiol. 2016, 7, 670. [CrossRef]

29. Gómez, E.; Ledbetter, C.A.; Hartsell, P.L. Volatile Compounds in Apricot, Plum, and Their Interspecific Hybrids. J. Agric. Food Chem. 1993, 41, 1669-1676. [CrossRef]

30. Pietruszka, M.; Pielech-Przybylska, K.; Szopa, J.S. Synthesis of higher alcohols during alcoholic fermentation of rye mashes. Scientific bulletin of the Technical University of Lodz. Food Chem. Biotech. 2010, 2010, 74.

31. Oliveira, J.M.; Faria, M.; Sá, F.; Barros, F.; Araújo, I.M. C6-alcohols as varietal markers for assessment of wine origin. Anal. Chim. Acta 2006, 563, 300-309. [CrossRef]

32. Etschmann, M.; Bluemke, W.; Sell, D.; Schrader, J. Biotechnological production of 2-phenylethanol. Appl. Microbiol. Biotechnol. 2002, 59, 1-8. [CrossRef] [PubMed]

33. Ohimain, E.I. Methanol contamination in traditionally fermented alcoholic beverages: The microbial dimension. Springerplus 2016, 5, 1-10. [CrossRef] [PubMed]

34. Miljić, U.; Puškaš, V.S. Influence of fermentation conditions on production of plum (Prunus domestica L.) wine: A response surface methodology approach. Hem. Ind. 2014, 68, 199-206. [CrossRef] 
35. Gómez-Plaza, E.; Ledbetter, C. The Flavor of Plums. In Handbook of Fruit and Vegetable Flavors; Wiley: Hoboken, NJ, USA, 2010; pp. 415-430, ISBN 9780470227213.

36. Miljić, U.; Puškaš, V.; Vučurović, V. Investigation of technological approaches for reduction of methanol formation in plum wines. J. Inst. Brew. 2016, 122, 635-643. [CrossRef]

37. Nunes, C.; Coimbra, M.A.; Saraiva, J.; Rocha, S.M. Study of the volatile components of a candied plum and estimation of their contribution to the aroma. Food Chem. 2008, 111, 897-905. [CrossRef]

38. Belitz, H.-D.; Grosch, W.; Schieberle, P. Food Chemistry; Springer: Berlin, Germany, 2004.

39. Zhao, N.; Zhang, Y.; Liu, D.; Zhang, J.; Qi, Y.; Xu, J.; Wei, X.; Fan, M. Free and bound volatile compounds in 'Hayward' and 'Hort16A' kiwifruit and their wines. Eur. Food Res. Technol. 2020, 246, 875-890. [CrossRef]

40. Blagoeva, N.; Bazhlekova, I.; Spasov, H.; Kostov, G. Influence of enzyme maceration and alcoholic fermentation temperature on the terpenes concentration in Muscat wine distillates. Bulg. J. Agric. Sci. 2020, 26, 1069-1075.

41. Stork, G.; Grieco, P.A.; Gregson, M. Allylic Chlorides from Allylic Alcohols: Geranyl Chlo-ride. In Organic Syntheses; John Wiley and Sons, Inc.: Hoboken, NJ, USA, 1974; p. 638.

42. Carballo, J. The Role of Fermentation Reactions in the Generation of Flavor and Aroma of Foods. In Fermentation. Effects on Food Properties; CRC Press: Boca Raton, FL, USA, 2016; p. 74. 